Published in final edited form as:

J Phys Chem B. 2020 October 29; 124(43): 9513-9525. doi:10.1021/acs.jpcb.0c07730.

\title{
Unusual Spectroscopic and Electric Field Sensitivity of Chromophores with Short Hydrogen Bonds: GFP and PYP as Model Systems
}

\author{
Chi-Yun Lin, \\ Department of Chemistry, Stanford University, Stanford, California 94305, United States \\ Steven G. Boxer \\ Department of Chemistry, Stanford University, Stanford, California 94305, United States
}

\begin{abstract}
Short hydrogen bonds, with heavy-atom distances less than $2.7 \AA$, are believed to exhibit proton delocalization, and their possible role in catalysis has been widely debated. While spectroscopic and/or structural methods are usually employed to study the degree of proton delocalization, ambiguities still arise, and no direct information on the corresponding potential energy surface is obtained. Here, we apply an external electric field to perturb the short hydrogen bond(s) within a collection of green fluorescent protein S65T/H148D variants and photoactive yellow protein mutants, where the chromophore participates in the short hydrogen bond(s) and serves as an optical probe of the proton position. As the proton is charged, its position may shift in response to the external electric field, and the chromophore's electronic absorption can thus reflect the ease of proton transfer. The results suggest that low-barrier hydrogen bonds (LBHBs) are not present within these proteins even when proton affinities between donor and acceptor are closely matched. Exploiting the chromophores as precalibrated electrostatic probes, the covalency of short hydrogen bonds as a nonelectrostatic component is also revealed. A theoretical framework is developed to address a possible contribution of unusually large polarizabilities of short hydrogen bonds due to proton delocalization, but no clear evidence for this phenomenon is found in accordance with the absence of LBHBs.
\end{abstract}

\section{Graphical Abstract}

\footnotetext{
Corresponding Author: Steven G. Boxer - sboxer@stanford.edu. Supporting Information

The Supporting Information is available free of charge at https://pubs.acs.org/doi/10.1021/acs.jpcb.0c07730.

Detailed experimental procedures, including sample preparation, spectroscopic methods, and X-ray crystallography; further technical discussion on related topics, such as structural analysis, Stark analysis, preresonance Raman analysis, and elaboration on theoretical models for proton polarizabilities; additional experimental data in figures and tables (PDF)

Complete contact information is available at: https://pubs.acs.org/10.1021/acs.jpcb.0c07730

The authors declare no competing financial interest.
} 


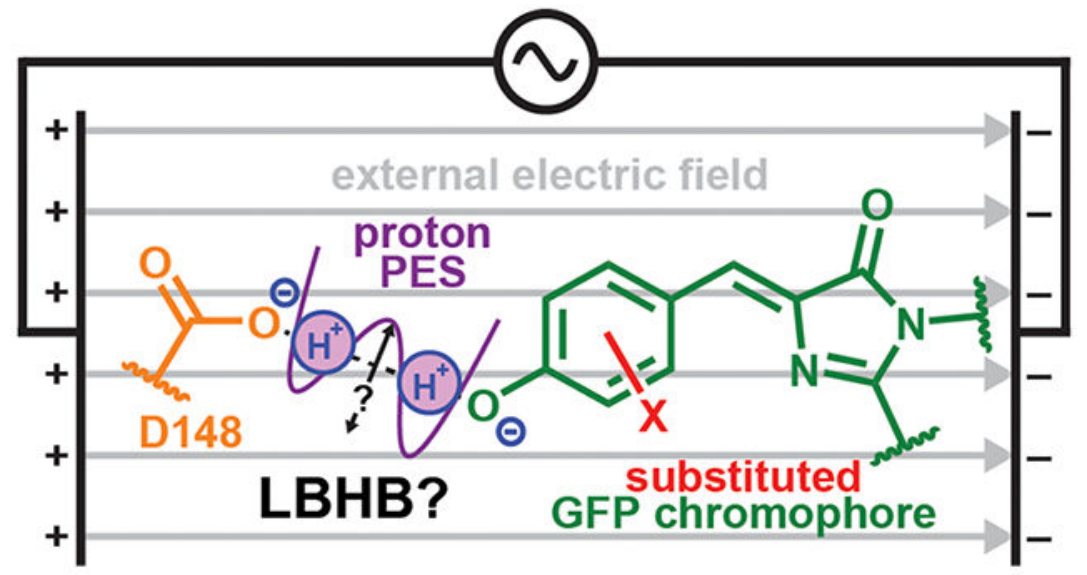

\section{INTRODUCTION}

Hydrogen bonds are arguably one of the most important chemical bonds and are ubiquitous in biomolecules and materials. ${ }^{1,2}$ Their intermediate strengths bridge between typical covalent and other noncovalent interactions, ${ }^{3}$ and they also play essential roles in mediating proton transfers. ${ }^{4}$ Research on hydrogen bonds has flourished over nearly a century ${ }^{5-7}$ since Linus Pauling first elucidated the nature of hydrogen bonds in the late $1930 \mathrm{~s} ;{ }^{8}$ nonetheless, the exact correlation between geometries and energetics of hydrogen bonds remains controversial. ${ }^{9,10}$ It is generally accepted that the topologies of potential energy surfaces (PESs) of protons in hydrogen bonds are strongly dependent on the heavy-atom distances $\left(R\right.$ 's) and the relative proton affinities $\left(\Delta \mathrm{p} K_{a}\right.$ 's) between donors and acceptors. ${ }^{11-13}$ Note that $\mathrm{p} K_{a}$ (also proposed under the name $\mathrm{p} K_{\mathrm{N}}{ }^{14}$ ) is used here to describe the proton affinity of buried residues instead of the more commonly used $\mathrm{p} K_{\mathrm{a}}$, which is complicated by water solvation. ${ }^{15}$ Because the proton is relatively light, nuclear quantum effects such as tunneling and delocalization can also be important especially for short hydrogen bonds, ${ }^{13,16}$ so this is a rich area of investigation.

The PESs for hydrogen bonds with heavy-atom separations ( $R$ 's) less than $2.7 \AA$ are especially difficult to generalize and can only be examined on a case-by-case basis; the placement of the zero-point energy (ZPE) with respect to the barrier between wells is hotly debated. ${ }^{11,17-20}$ If the proton affinity on each side is mismatched, one would expect the proton to be localized on the donor, and the hydrogen bond is classified as a strong ionic hydrogen bond (SIHB). ${ }^{21}$ When the proton affinities of donors and acceptors are closely matched, ${ }^{12}$ the PESs become shallow and strongly anharmonic due to the strong coupling between the proton binding sites. ${ }^{13}$ This could bring the ZPE close to or above the barrier and result in low-barrier hydrogen bonds (LBHBs), in which the proton is delocalized between donors and acceptors, and the corresponding PES is virtually indistinguishable from a single-well potential. Otherwise, the proton is equally likely to localize on the donor or acceptor, and we have a double-well potential. ${ }^{17}$ Owing to the abundance and hypothesized strengths of short hydrogen bonds in proteins, especially at the active sites of many enzymes, ${ }^{22,23}$ the functional contribution of LBHBs to the catalytic power of enzymes has been actively debated. ${ }^{24,25}$ The search for functionally important LBHBs in proteins 
has been rather difficult, however, ${ }^{26-28}$ since no single approach can provide unambiguous evidence for the degree of delocalization. ${ }^{19} \mathrm{X}$-ray and neutron diffraction are utilized to characterize the nuclear coordinates, while energetic information is mostly extracted from spectroscopic studies frequently combined with isotope substitution. ${ }^{2,10,29-33}$

In our previous work, a short hydrogen bond with the hydroxyl group of the protonated neutral or A state of the chromophore was discovered in a particular green fluorescent protein (GFP) mutant, S65T/H148D, using X-ray crystallography (Figure 1A). ${ }^{34}$ This was accompanied by an unusually featureless visible absorption band at $77 \mathrm{~K}$ compared with that of the normal A state of the chromophore when histidine is at position $148 .{ }^{35} \mathrm{We}$ recently systematically tuned the chromophore's $\mathrm{p} K_{a}$ via halogenation (Figure 1B,C) to test whether nearly zero $\Delta \mathrm{p} K_{a}$ and short $R$ between the chromophore and D148 are sufficient for a LBHB to exist. ${ }^{36}$ To characterize the energetics of the short hydrogen bond across the halogenated series, the spectral isotope effect (SIE) together with isotope fractionation factor at room temperature were measured by exploiting the halogenated chromophore as both an active participant in the short hydrogen bond and a sensitive optical probe of the proton position. The short $\mathrm{O}-\mathrm{O}$ distance was demonstrated to persist throughout the variant series, but the data were inconsistent with an LBHB despite the close donor-acceptor proton affinity. In the present work, we extend the variant series (Figure 1C) and use electronic Stark spectroscopy at $77 \mathrm{~K}$ to provide new insights. ${ }^{37}$ Because proton transfer involves the movement of charge, one expects that it could be sensitive to an electric field, whether from the protein itself or an applied field. Thus, Stark spectroscopy can provide a novel approach for analyzing the extent of proton transfer between two wells and the degree of proton delocalization can be inferred, quite analogous to our previous applications to electron delocalization in mixed-valence systems. ${ }^{38}$

Electronic Stark spectroscopy can also serve as a useful tool for extracting the underlying populations from the broad visible absorption bands associated with the S65T/H148D variants based on differences in Stark tuning rates. We associate the deconvolved populations with the proton being in each well, corresponding to a state with the protonated chromophore possessing a slightly lengthened $\mathrm{O}-\mathrm{H}$, which we will call an "A-like A state", and another state with the deprotonated chromophore engaging in a short hydrogen bond with its protonated partner D148, denoted a "B-like A state" (the B state of the GFP chromophore is the deprotonated form) (Figure 1B). We find that the correlations between the Stark tuning rates and the absorption maxima deviate from the calibration curves obtained through mutants with a normal hydrogen bond to the chromophore, i.e., not involving the H148D mutation, ${ }^{39,40}$ suggesting the effect of the short hydrogen bond on the chromophore cannot be solely explained by electrostatics as was the case for these species with normal hydrogen bonds. Rather, it is likely that the covalency of the short hydrogen bond, owing to extended electron delocalization, alters the electronic properties of the neutral and deprotonated chromophores. Replacement of exchangeable protons with deuterons is also conducted to fine-tune the $\mathrm{O}-\mathrm{H}(\mathrm{D})$ distance and gauge the influence of the proton (deuteron). To complement the results from the short-hydrogen-bond GFPs, photoactive yellow protein (PYP) and some of its mutants, which were previously characterized to be a closely related system ${ }^{39}$ and possess two short hydrogen bonds with its chromophore (Figure 2), ${ }^{18-20,41,42}$ are also analyzed and help strengthen the findings. 
This study points out the fundamental differences between short hydrogen bonds, which have non-negligible covalency, 2,30,31,43,44, and other noncovalent interactions (e.g., normal hydrogen bonds, $\pi$ stacking, and hydrophobic interactions), which can be adequately described by classical electrostatics. ${ }^{45}$ We also shed light on the coupling between a $\pi$-conjugated system and short hydrogen bonds, which is of particular interest for understanding and designing molecular assemblies in the fields of resonance-assisted hydrogen bonds (RAHBs) ${ }^{12,14}$ and hydrogen-bond-mediated mixed-valence systems. ${ }^{46,47}$

\section{EXPERIMENTAL METHODS}

The experimental methods, including sample preparations, UV-vis absorption measurements, 77 K Stark spectroscopy, preresonance Raman spectroscopy, and X-ray crystallography, are detailed in the Supporting Information Sections S1-S3.

\section{RESULTS AND DISCUSSION}

\subsection{Characterization of Short Hydrogen Bonds and Their Proton PESs.}

3.1.1. Mutant and Variant Design.-We introduced three more members, Y66(2,3$\left.\mathrm{F}_{2} \mathrm{Y}\right), \mathrm{Y} 66\left(3-\mathrm{Br}_{1} \mathrm{Y}\right)$, and $\mathrm{Y} 66\left(3-\mathrm{I}_{1} \mathrm{Y}\right)$, to the series of GFP chromophore variants (Figure 1C) in the background of S65T/H148D (short-hydrogen-bond GFP) ${ }^{36}$ to better match the proton affinities between the donor and acceptor (Table 1), as determined by titrating the chromophores under denaturing condition (Figure S24). We chose not to approximate the proton affinity of the substituted chromophore with the measured $\mathrm{p} K_{\mathrm{a}}$ in the native protein environment, as the presence of the short hydrogen bond would likely perturb the $\mathrm{p} K_{\mathrm{a}}$ of the chromophore to different degrees across the halogenated variant series. The chromophore could also only undergo proton exchange with the bulk solvent through hydrogen-bond relays rather than be directly solvent accessible. X-ray crystallography revealed a consistent $\mathrm{O}-\mathrm{O}$ distance of 2.4-2.6 $\AA$ for the short hydrogen bond across the variants within experimental error (Table 1 and Table S8, see also Figure S6). It is not possible to assign the proton position even with the highest-resolution $\mathrm{X}$-ray structure at hand $\left(\mathrm{Y} 66\left(2,3-\mathrm{F}_{2} \mathrm{Y}\right), 1.18 \AA\right.$ ), but on the basis of extensive empirical observations on short hydrogen bonds ${ }^{2,30}$ and theoretical modeling, ${ }^{13,36}$ we can safely assume that the proton equilibrium position is slightly shifted away from either the proton donor or acceptor compared to the normal hydrogen bond. In particular, if the chromophore acts as a proton donor (i.e., having a larger $\mathrm{p} K_{a}$ than its partner D148), the $\mathrm{O}-\mathrm{H}$ distance is estimated to lengthen from $\sim 0.95$ to $\sim 1.05 \AA$ due to the stronger coupling between the donor and acceptor O-H potentials. ${ }^{13,36}$

PYP serves as another superb model system for elucidating the influence of short hydrogen bonds on chromophore properties. It is known from the crystal structures of wild-type Halorhodospira halophila PYP (HhPYP) that the chromophore, anionic p-coumaric acid, interacts with its neighboring residues Y42 and E46 through two short hydrogen bonds (Figure 2, Table 1). In addition to the thoroughly scrutinized HhPYP, we also choose to study PYP from Salinibacter ruber (SrPYP), ${ }^{51}$ which has been found to exhibit larger SIEs at room temperature than HhPYP. ${ }^{52}$ Even though no crystal structure has been solved for SrPYP to date, the conserved Y42 and E46 from sequence alignment and the large SIE 
suggest the existence of at least one short hydrogen bond between the chromophore and these residues. In addition to the PYPs from two different species, we include a combination of HhPYP Y42F and E46Q mutations to break the hydrogen bond associated with the former and/or elongate that associated with the latter (Figure 2, Table 1) to probe the effect of short hydrogen bonds. ${ }^{41,49,50}$ Unfortunately, SrPYP is effectively negatively supercharged (nearly 20\% aspartate and glutamate), such that the Y42F mutation leads to chromophore protonation by raising the chromophore's proton affinity and breaking the short hydrogen bond(s) (Figure S13).

Note that we use "variants" and "mutants" to distinguish changes to the chromophore introduced by amber suppression and protein environment, respectively, to facilitate the following discussion. As a useful shorthand, "anomalous A state" refers to a chromophore that engages in short hydrogen bond(s) $(R<2.7 \AA)$, as in the S65T/H148D GFP variants and PYP mutants, while "normal A state" is reserved for neutral chromophores that only participate in normal hydrogen bond(s) $(R>2.7 \AA)$ or other noncovalent interactions, such as those in the $\mathrm{H} 148$ chromophore variants. For clarity, since there is one proton present between D148 and the chromophore in these short-hydrogen-bond GFPs (Figure 1B), rather than referring to these two species as the conventional A and B states, we shall designate these species as A-like and B-like A states, the population ratio of which is internally governed by $\Delta \mathrm{p} K_{a}$ instead of external $\mathrm{pH}^{53}$ We reserve the actual B state to the case in which both D148 and the chromophore are deprotonated at high $\mathrm{pH}$, which is still redder than the red band (B-like A state) deconvolved from absorption of the anomalous A state (see below). In other words, the hydrogen bond is no longer present between D148 and the chromophore in the B state because there is no mediating proton; in fact, D148 flips away from the chromophore as determined by X-ray crystallography (see Section S6 in ref 36). The analogous A-like A state is absent in PYPs because of the large $\mathrm{p} K_{a}$ mismatch between the PYP chromophore and E46 or Y42, ${ }^{18,20,42,52}$ resembling the case of the 3,5- $\mathrm{Cl}_{2} \mathrm{Y}$ H148D GFP variant, so no further deconvolution is required for PYPs, as also suggested by Stark spectroscopy (Section S5, see also ref 54).

\subsubsection{Proton PESs and Deconvolution of Short-Hydrogen-Bond Protein Electronic Absorption Spectra by Stark Spectroscopy.- ${ }^{1} \mathrm{H}$ NMR would be a} superb method for probing the short hydrogen bonds due to the proton's expected downfield chemical shift. ${ }^{10,20}$ However, no downfield signal was found for these short-hydrogen-bond GFPs (Section S7 of ref 36; see also ref 34), presumably due to the sought-after protons being in chemical exchange with solvents at an unfavorable time scale. ${ }^{56}$ Instead, we analyze these short hydrogen bonds with other spectroscopic methods at hand. Unlike the $77 \mathrm{~K}$ electronic absorption spectra for most normal A states, ${ }^{40}$ the spectra for the A states from H148D GFP variants remain relatively featureless in a frozen glass at low temperature but exhibit a consistent dip in the middle except for the Y66 counterpart (Figure 3A and Figure S26). If the associated short hydrogen bond corresponds to a LBHB, the spectral dip could be the vibronic feature of absorption from a single species with a delocalized proton. However, deuteration not only widens the absorption band but also enhances the dip (Figure $3 \mathrm{~A}$ and Figure S26), especially for variants with nearly zero $\Delta \mathrm{p} K_{a}$ (Figure 3B). 
When the Stark tuning rates are different for underlying populations, the Stark spectra, whose line shapes are typically dominated by the second derivative of the absorption, are very useful for deconvolving the bands (Section S5). Through simultaneous fitting of both low-temperature absorption and Stark spectra, one homogeneous population with only one set of electro-optic parameters is insufficient to account for features across the entire absorption and Stark spectra (Figure 3C and Figure S9), justifying the assertion that there are at least two populations. This is rather unusual, as the absorption and Stark spectra of normal GFPs in either deprotonated ${ }^{39}$ or protonated states ${ }^{40}$ can be fully accounted for with a single set of electro-optic parameters shared between the main and vibronic sidebands. Assuming two populations and constraining with minimal spectral overlap, two distinct bands are resolved for all variants (Figure 3C and Figure S9), except for Y66 and $\mathrm{Y} 66\left(3,5-\mathrm{Cl}_{2} \mathrm{Y}\right)$ variants due to the larger degrees of $\mathrm{p} K_{a}$ mismatch (Table 1). Interestingly, the redder band resembles the typical B-state band shape found in many normal GFPs, ${ }^{39}$ and its vibronic feature contributes to the dip. The bluer band is broad and featureless, behaving more similarly to the absorption band of the H148D Y66 variant than the vibronic structure observed in most other normal A-state spectra at low temperature. ${ }^{40}$ On the basis of the population ratio and $\Delta \mathrm{p} K_{a}$ (Figure S9), the red and blue bands can be intuitively assigned to species with the proton localized in each well of the PES. This is consistent with and reinforces the conclusion from the previous room-temperature study. ${ }^{36}$ This assignment also agrees with the trend of SIE for each species: the underlying red band red-shifts and the blue band blue-shifts upon deuteration (Figure 3A and Figure S26, Table S10). This SIE can be explained by the anharmonicity of the double-well PES causing a larger tendency for the deuteron to localize toward the donor or acceptor compared to the proton (Figures 1B and $3 \mathrm{~B}$ ), and these subtle changes in proton or deuteron positions can be sensitively probed by the chromophore absorption spectra. Population transfer between two wells caused by the external electric field should manifest as zeroth derivatives (a "non-classical" Stark effect ${ }^{57}$ ) rather than the typical second-derivative line shapes from charge displacement upon excitation (a linear Stark effect; ${ }^{37}$ see Section S5 for more discussion). Such a zeroth derivative component is not observed for each band in the Stark spectra within our ability to deconvolve the data (Figure 3C and Figure S9), suggesting that external electric field driven proton transfer is not significant, and hence, there is a high barrier in the PESs. This can be further attested by the $4 \omega$ (where $\omega$ is the field modulation frequency, see ref 37) spectra resembling second derivatives of $2 \omega$ spectra (Figure S11), as expected for charge displacement upon excitation rather than proton transfer within the short hydrogen bond. ${ }^{37}$

Besides the sum-of-derivative analysis of the Stark spectra that has been discussed so far, the field strength $F_{\text {ext }}$ dependence of Stark spectra (both $2 \omega$ and $4 \omega$ ) provides additional qualitative evidence for the proposed topology of the proton PES. Significant deviation from the typical external field dependence of Stark spectra (i.e., $2 \omega$ and $4 \omega$ spectra scaling with $F_{\text {ext }}^{2}$ and $F_{\text {ext }}^{4}$, respectively) observed for charge displacement upon excitation is expected for borderline single-well/double-well cases due to proton transfer through the low barrier between the wells (Section S5; ref 57), while a single-well potential still follows the classical field dependence as the proton is extensively delocalized. The absence of deviations for all variants (Figure S12) suggests that either population exchange between two wells through tunneling and thermal activation is minimal at $77 \mathrm{~K}$ or the currently achievable 
strength for the applied electric field is still too low for significant external field-induced proton transfer to occur. Applied fields of $\sim f \times 1.4 \mathrm{MV} / \mathrm{cm}$ can be achieved on frozen glasses before dielectric breakdown, where $f$ is the local field factor, which is necessary due to the larger field experienced by the chromophore compared to the externally applied field based on the unavoidable polarization effect of the chromophore environment. ${ }^{37}$ Either way, this rules out the existence of a LBHB in the S65T/H148D variants. A single-well potential for the short hydrogen bond could also be inferred just on the basis of the observed field dependence and the second-derivative line shapes, but this scenario is ruled out in combination with precedent evidence from the inadequacy of one-band fit for lowtemperature Stark spectra (Figure 3C and Figure S9) and room-temperature SIE/isotope fractionation factors. ${ }^{36}$

\subsubsection{Proton PESs and Deconvolution of Short-Hydrogen-Bond Protein Vibrational Spectra by Raman Spectroscopy.-To further establish the topology of} the PES, we also acquired the corresponding room-temperature vibrational spectra of the chromophore's phenol stretching mode (around 1230-1270 $\mathrm{cm}^{-1}, 58,59$ see also Section S6) for the S65T/H148D variants using preresonance Raman spectroscopy (Figure 4A). This method combines the advantages of selectively enhancing signals from vibrational modes that are strongly coupled to the chromophore excitation (in contrast to IR spectroscopy), showing narrow peak widths (as opposed to UV-vis spectroscopy), and possessing a fast intrinsic time scale comparable to that of electronic transitions (unlike IR and NMR spectroscopy). The phenol stretching mode blue-shifts as the chromophore becomes deprotonated ${ }^{58,59}$ and is therefore a sensitive proton probe. If the population exchange between A-like and B-like A states is much slower than the electronic time scale, a split in the phenol stretching peak would be expected, provided that the peak width is narrow enough to be resolvable. This is reminiscent of the spectroscopic strategies exploited to determine the $\Delta \mathrm{p} K_{a}$ of a catalytically relevant hydrogen bond in ketosteroid isomerase $(\mathrm{KSI})^{60}$ and the degree of electron delocalization in 2-norbornyl cation ${ }^{61-63}$ and mixedvalence compounds. ${ }^{64-67}$ While the Raman spectra of the protonated $3-\mathrm{Cl}_{1} \mathrm{Y}, 3-\mathrm{Br}_{1} \mathrm{Y}$, and $3-\mathrm{I}_{1} \mathrm{Y}$ variants show a single peak, two peaks can be readily seen upon deuterium exchange (Figure 4A and Figure S19), indicating a placement of the deuteron ZPE below the barrier between two wells of the hydrogen-bond PES and setting a nonzero lower bound on the barrier height (Figure 4B). No conclusive placement of the proton ZPE can be inferred if we solely rely on the spectra for the protonated species. However, Raman features from both protonated and deuterated samples can be well-interpreted on the basis of the ground-state wave functions of proton and deuteron within the PES calculated from McKenzie's onedimensional coupled Morse potential model, ${ }^{13,36}$ in which the proton wave function is still fairly delocalized even with its ZPE below the barrier and may be the reason for the merging of the two underlying peaks (Figure 4B). Note that the calculated PESs suggest a tunneling splitting (i.e., the energy difference the ground and first excited states) of $\sim 700 \mathrm{~cm}^{-1}$, which implies a lack of significant population for the proton in the first excited state even at room temperature $\left(k_{\mathrm{B}} T \sim 200 \mathrm{~cm}^{-1}\right)$. As such, conclusions from both room-temperature and $77 \mathrm{~K}$ measurements should be comparable so long as the geometries are consistent. The peak(s) of interest for the even better $\mathrm{p} K_{a}$-matched fluorinated variants are unfortunately masked 
by the intense features from C-F stretching (Section S6, Figure S21), and thus no useful information could be obtained from these constructs.

\subsection{Perturbation of the Short Hydrogen Bonds.}

The strategy of modulating $\Delta \mathrm{p} K_{a}$ via chromophore halogenation and using the chromophore absorption as a proton reporter is a double-edged sword: this approach is minimally perturbative in terms of structures (however, see Section S4 for more discussion on structures), yet the color of the chromophore can be affected by both the proton position and effects due to substituent-specific modulations in electronic distribution. Both are simultaneously changed by the substituents and therefore hard to tease apart. Closer examination of the absorption maxima from the deconvolved bands reveals that while the trend in colors for both A-like and B-like A states across variants is primarily governed by the electronic distribution of the GFP chromophore, $\Delta \mathrm{p} K_{a}$ only affects the A-like A states significantly. ${ }^{12,31}$ Specifically, if we plot the B state absorption maxima for the corresponding H148 variants (Table S11) against the A-like and B-like A states' absorption maxima, clear correlations for both can be seen, especially for the latter (Figure 5A, dashed lines). H148 variants are chosen because D148 is found to be twisted to the exterior of the protein and no longer engages in hydrogen bonding with the chromophore in the B state (see Section S6 in ref 36), and B states are chosen because there is no normal A state to compare against for these halogenated chromophores due to their low $\mathrm{p} K_{\mathrm{a}}$ 's. However, if we plot the absorption maxima against $\Delta \mathrm{p} K_{a}$, a more evident trend can only be observed for the A-like A state (Figure 5B), which likely suggests that the modification in $\mathrm{O}-\mathrm{H}$ distances through $\Delta \mathrm{p} K_{a}$ tuning is not sufficient to dominate the electronic effect for the B-like A state. This is not surprising, since the proton is closer to the chromophore in the A-like A state (Figure 1B). We will see that the same phenomenon is at work when we later scrutinize the Stark tuning rates for both anomalous A states. In hindsight, it was fortunate in our previous study ${ }^{36}$ that the absorption maxima of the unresolved room-temperature anomalous A state bands from short-hydrogen-bond GFPs reflect the underlying population ratio from each well (Figure S25), and the resulting effect overwhelms the complicating electronic perturbation and allowed us to extract the direct consequences from $\Delta \mathrm{p} K_{a}$ tuning.

To study the influence of the short hydrogen bond on the chromophore's electronic structure, we extracted the Stark tuning rates for the population in each well of the PES from the protonated and deuterated S65T/H148D GFP variants (as in Figure 3C) and list them in Table 2. At first glance, other than the almost consistent and interesting decrease in Stark tuning rates upon deuteration, discussed in detail below, it is rather difficult to pinpoint an obvious trend as a function of $\Delta \mathrm{p} K_{a}$ due to the aforementioned convolution of proton position and electronic effects from the halogen substituents. This convolution can be resolved, however, by comparison to the deprotonated and protonated chromophores under electrostatic influences ${ }^{39,40}$ to isolate the additional perturbation of the short hydrogen bond from the electrostatic effect. This approach also relieves the severe problem of comparing H148D species with their H148 counterparts (Figure 5A), as both changes in hydrogen bond distances and hydrogen bond partners are accounted for by the electrostatic responses of the calibrated chromophore. 
We examine the B-like A state first (Figure 6A). The red line in Figure 6A is the absorption maximum vs Stark tuning rate correlation curve for the unsubstituted normal B state anionic chromophore to quantitatively capture its electrostatic response. This curve is a fit from the data of S65T environmental mutants using the Marcus-Hush model and diabatic states in Figure 6D (left) developed in our previous work. ${ }^{39}$ We have previously demonstrated that it is meaningful to treat chromophore variants and environmental mutants the same way in terms of electron density modulation ${ }^{39}$ (see also ref 68 ), so we can safely perform the same analysis here even with the change in chromophore identities. Data from H148 with halogenated chromophore variants are also shown in squares. As previously detailed in ref 39, while the variants follow a general trend consistent with the fitted curve from environmental mutants, some non-negligible deviations from the environmental mutants can be observed, reflecting characteristics from individual substituted chromophores and complicating the use of these chromophore variants as consistent electrostatic probes. We expect that the same deviation also occurs in the B-like A state for H148D variants. Indeed, as we plot the corresponding data in Figure 6A, the same general pattern persists, albeit with bluer absorption maxima and larger Stark tuning rates. On the other hand, no obvious trend related to $\Delta \mathrm{p} K_{a}$ can be identified (as opposed to the A-like A state, vide infra), and if a trend does exist, it is likely masked by the substitution-specific electronic effect. Data from H148D variants exhibit a larger spread compared to the H148 counterparts (Figure $6 \mathrm{~A})$. Both data sets also noticeably biased toward the side of larger $f \Delta \mu_{\mathrm{CT}}$ or smaller $V_{0}$ within the framework of the Marcus-Hush treatment (Figure 6D, left). ${ }^{39}$ At this point, it is not possible to distinguish which of the two Marcus-Hush parameters, $f \Delta \mu_{\mathrm{CT}}$ and $V_{0}$, is affected more by the short hydrogen bond because of the intrinsic electronic variations due to substituents on the chromophores.

To help clarify and resolve this GFP chromophore variant complexity, HhPYP mutants can offer an incisive answer, since they exist in a B-like A state and possess the same chromophore but various hydrogen-bonding patterns through mutagenesis at Y42 and E46 (Table 1 and Figure 2). We have concluded in our previous work ${ }^{39}$ that, by comparing the correlation between the Stokes shift and absorption maximum from PYP and GFP mutants, both anionic chromophores follow the same quantitative behavior and share the same electronic coupling $V_{0}$ even in the presence of short hydrogen bonds. In contrast, when analyzing with the correlation plot between the absorption maximum and Stark tuning rate (Figure 6B), as we alter the number of short hydrogen bonds in HhPYP from two (Y42/E46) to one (Y42/E46Q and Y42F/E46) and finally to zero (Y42F/E46Q), the data point moves closer and closer to the GFP-based B-state fit curve and finally sits exactly on it. This shows an unambiguous trend of decreasing $f \Delta \mu_{\mathrm{CT}}$ or increasing $V_{0}$ as we remove more short hydrogen bonds. Because we know that $V_{0}$ is unchanged across this mutant series, ${ }^{39} f \Delta \mu_{\mathrm{CT}}$ becomes the only factor that is influenced by the short hydrogen bond. By extension, we can argue that the S65T/H148D GFP variants likely share the same behavior, but it is concealed by the additional substituent-specific electronic effects from halogenation. Conversely, the SrPYP mutants exhibit the opposite trend upon E46Q mutation, which is different from that expected from the room-temperature SIE study, ${ }^{52}$ suggesting a nontrivial interplay between the two putative short hydrogen bonds that could be studied through NMR. ${ }^{69}$ 
For the A-like A state, the same exercise can be duplicated (Figure 6C). The A-state fit curve $^{40}$ is exploited to reflect the electrostatic response of the unsubstituted normal $\mathrm{A}$ state chromophore. Deviations of H148D variants from the A-state fit curve can again be seen, but this time they correspond to larger $V_{0}{ }^{\prime}$ or smaller $f_{\mu \mathrm{CT}}$ than the normal A state according to the three-form model (Figure 6D, right). ${ }^{40}$ Since there is no valid comparison for the A-like A state with the corresponding halogenated chromophores in the normal A state due to the low $\mathrm{p} K_{\mathrm{a}}$ 's of halogenated chromophores, we cannot rule out the possibility of substituent-specific electronic effects to explain the absence of any obvious pattern of these data points. However, the fact that values with larger deviations correspond to better $\mathrm{p} K_{\alpha}$-matched variants suggests that the electronic structure of the protonated chromophore is modulated through the proton when it serves as a proton donor in a short hydrogen bond. Relying on the robust insensitivity of $V_{0}$ for the deprotonated halogenated chromophore from the Stokes shift study ${ }^{39}$ and the previous conclusion from the B-like A state, we speculate that $f \mu_{\mathrm{CT}}$ could again be the only parameter that is subject to the perturbation of the short hydrogen bond. Just as how we scrutinized the variation in $V_{0}$ of the deprotonated chromophore through its Stokes shift, this claim could in principle be tested with the correlation of Stokes shift from $A^{*}$ emission and absorption maximum (as in Figure 8 in ref 39) by considering an additional vibronic coupling, but the relevant measurements are so far inaccessible for most GFP mutants and variants given the efficiency of excited-state proton transfer and the chromophore's low $\mathrm{p} K_{\mathrm{a}}$.

In summary, we have identified that $f \Delta \mu_{\mathrm{CT}}$ for the deprotonated chromophore and $f_{\mu \mathrm{CT}}$ for the protonated chromophore increases and decreases, respectively, in the presence of short hydrogen bond(s). Both quantities are products of the (difference) dipole moment $(\Delta) \mu_{\mathrm{CT}}$ and the local field factor $f$, which arise from the electron distribution of the $\pi$ system per se and the polarizability of the $\pi$ system's environment, respectively. In the following, we will propose a mechanism for the short hydrogen bond to perturb each of the quantities and examine the corresponding plausibility.

3.2.1. Perturbation on Dipole Moments.-On the basis of the short hydrogen bond's ability to modulate the $\mathrm{O}-\mathrm{H}$ internuclear distance, the (difference) dipole moments of the chromophores, which are of electronic origin, can be evidently tuned by the short hydrogen bond. Specifically, the chromophore's O-H distance is lengthened or shortened when the chromophore behaves as a proton donor or acceptor, respectively. ${ }^{30,31}$ We have asserted in our previous work that the A state behavior is not at all akin to the B state counterpart owing to the covalency of the $\mathrm{O}-\mathrm{H}$ bond, ${ }^{40}$ so we should expect a deviation in the character of the A-like A state from an electrostatically perturbed normal A state. By elongating the $\mathrm{O}-\mathrm{H}$ bond, we expect the CT form (Figure 6D, right) to be less charge localized on both ends of the chromophores, thereby reducing $\mu_{\mathrm{CT}}$ (Figure 6E, right).

To understand the B-like A state's response to the short hydrogen bond, we need to invoke the covalent nature of the short hydrogen bonds to explain the deviation from the electrostatically modulated B state, which includes examples perturbed by normal hydrogen bonds. ${ }^{39}$ The covalency of short hydrogen bonds as opposed to normal ones has been documented with NMR. ${ }^{70}$ It results from the delocalization of electrons between the proton donor and acceptor, where the exchange interaction is necessary for the short hydrogen 
bond to overcome the unfavorable van der Waals repulsion between the overlapping heavy atoms of the proton donor and acceptors, and so a model with only classical electrostatics is insufficient to predict the existence of short hydrogen bonds. ${ }^{43}$ This also rationalizes the inability of using classical molecular dynamics to simulate and maintain the short hydrogen bond (O-O distance $2.6 \AA$ ) between Y16 of KSI and its substrate analogue 19-nortestoterone (Supplementary Text 4 from ref 71) in contrast to QM/MM simulations in ref 72. In the language of molecular orbitals, the lone pair electrons in the nonbonding orbital of the proton acceptor partially occupies the vacant $\sigma^{*}$ orbital of the donor-proton covalent bond, ${ }^{31,43,44}$ resulting in a more delocalized electron distribution within the short hydrogen bond and a weakening and lengthening of the donor-proton bond (Figure 6E, left). The interaction strength strongly depends on the energy matching of the two involved orbitals, which is equivalent to $\mathrm{p} K_{\mu}$ matching. Therefore, when the chromophore behaves as a proton acceptor in the B-like A state, it donates its electrons and leads to a more spread-out electron distribution, and hence a larger $\Delta \mu_{\mathrm{CT}}$.

By virtue of forming a short hydrogen bond with its partner, the number of electrons within the chromophore is no longer conserved due to electron delocalization across the hydrogen bond. A similar phenomenon can be achieved when the chromophore is covalently modified by halogens, as the identity of the chromophore becomes slightly different. Since the proton is attached to the chromophore through a true covalent bond (a hydroxyl group) rather than a partially covalent hydrogen bond, the A-like A state is more sensitive to changes in $\Delta \mathrm{p} K_{a}$. In contrast, the B-like A state is understandably dominated by the electronic effect from substitutions, yet another covalent interaction, in the chromophore variants. This is reminiscent of the effect on the absorption maxima observed in Figure 5. The phenomenon of short hydrogen bonds modulating $\pi$ systems has also been extensively investigated, notably in the context of RAHB ${ }^{12,14}$ and hydrogen-bond-mediated mixedvalence complexes, ${ }^{46,47}$ in which electrons within hydrogen bonds can be thought of as part of the delocalized $\pi$ systems. ${ }^{14}$

3.2.2. Perturbation on Local Field Factors.-In addition to the change in electronic dipole moments discussed so far, another provocative proposal is that proton polarization within the short hydrogen bond(s) might manifest itself as a local field factor $f$, as this could also increase $f \Delta \mu_{\mathrm{CT}}$ for the B-like A state. While we will argue below that this is likely not making a significant contribution, the underlying concepts are interesting and may apply to other short hydrogen bond systems, so the basic idea is developed in the following and in further detail in Sections S7 and S8. The local field factor is typically considered as originating from field-induced molecular rotation of solvent (the reaction field), nuclear displacement, and electronic distortion in the environment. We are not aware of any analysis suggesting that $f$ could also specifically be affected by electric-field-induced proton polarization, which is analogous to magnetic-field-induced ring currents that account for the (de)shielding of nuclei in $\mathrm{NMR}^{73}$ (see footnote 100 in the Supporting Information). While we have shown in Section 3.1.2 that the proton in the short hydrogen bond of GFP variants cannot be transferred across wells via experimentally accessible external electric fields, it could be displaced slightly within each well. 
Treating the problem classically, since a proton carries a positive elementary charge $+e$, an external field $F_{\text {ext }}$ can shift its mean position, whose displacement $\Delta r$ is determined by the local curvature of the hydrogen bond PES, $V_{\mathrm{HB}}{ }^{\prime \prime}\left(r_{0}\right)$, and reflects the degree of proton delocalization (Figure 7, more in Sections S7 and S8):

$$
\Delta r=\frac{e F_{\mathrm{ext}}}{V_{\mathrm{HB}}{ }^{\prime \prime}\left(r_{0}\right)}
$$

where $r_{0}$ is the most probably position of the proton wave function in the absence of an applied field, and $\Delta r$ refers to the difference between situations with and without the external field. The displaced proton subsequently generates an induced dipole field $\Delta F_{\mathrm{HB}}$ and effectively amplifies the external field $F_{\text {ext }}$, leading to a larger internal field $F_{\text {int }}$ sensed by the GFP or PYP chromophore (Figure 7):

$$
\begin{aligned}
F_{\text {int }} & =F_{\text {ext }}+\Delta F_{\mathrm{HB}}+\Delta F_{\text {non-HB }} \\
& =\left[1+\frac{2 e^{2}}{4 \pi \epsilon_{0} r^{3}} \frac{1}{V_{\mathrm{HB}}{ }^{\prime \prime}\left(r_{0}\right)}+\left(f_{\text {non-HB }}-1\right)\right] F_{\text {ext }} \equiv f F_{\text {ext }}
\end{aligned}
$$

where $r$ is the distance between the proton and the phenol(ate) oxygen (Section S7), and $\epsilon_{0}$ is the vacuum permittivity. The induced fields from other sources are collectively denoted as $\Delta F_{\text {non-HB }}\left(=\left(f_{\text {non-HB }}-1\right) F_{\text {ext }}\right)$ and factored in according to the superposition principle, assuming they are not simultaneously affected by the polarized short hydrogen bond. Note that the overall local field factor $f$ reduces to $f_{\text {non-HB }}$ in the absence of a short hydrogen bond. Since the principal axes for the polarizable moieties are in general not colinear with externally applied fields, any local field factor should be a tensor rather than a scalar as shown in eq 2, and we reserve a more explicit tensor treatment for Section S7. The concept of proton polarizability was strongly advocated by Georg Zundel, who invoked the existence of such a notion for the $\mathrm{p} K_{\alpha}$-matched short hydrogen bond in the Zundel cation $\left(\mathrm{H}_{2} \mathrm{O} \cdots \mathrm{H}^{+} \cdots \mathrm{OH}_{2}\right)$ to explain the broad continuum IR band of diluted aqueous acid due to the susceptibility of the proton position to environmental electrostatic fluctuations. ${ }^{74}$ Along the same vein, Perrin and Lau showed that protons that are found to be equally shared between donors and acceptors of short hydrogen bonds in crystals could localize on either side owing to their sensitivity to the environment that leads to symmetry breaking, ${ }^{17,75}$ reminiscent of electron localization in the Creutz-Taube ion at $77 \mathrm{~K}^{38}$

On the basis of eq 2, the shallower the PES is, corresponding to shorter heavy-atom distances and/or better $\mathrm{p} K_{a}$ matching, ${ }^{13}$ the more delocalized the associated proton is and the more polarizable the hydrogen bond is, causing a larger local field factor $f$. Even though we argue that the PESs for the short hydrogen bond in the protonated S65T/H148D GFP variants are double-welled, the local curvatures at the energetic minima should be smaller than that of a normal hydrogen bond (Section S8). As the GFP chromophore can sense the delocalized proton within each well, smaller local curvatures could result in the relatively featureless electronic absorption bands observed from short-hydrogen-bond GFPs (Figure 3A). However, because the proton is closer to the chromophore for the A-like A state than the B-like A state, the former should have a larger $f$ than the latter (eq 2), which conflicts with the decrease in $f \mu_{\mathrm{CT}}$ due to the short hydrogen bond for the A-like A state, indicating 
that the proton polarizability could not be the major contributor to the anomaly in the observed Stark tuning rates.

Since $f$ is positively correlated with the degree of proton delocalization, a simple way to assess its existence is to measure the Stark tuning rates of proteins where protons are exchanged for deuterons. The consistent decrease in Stark tuning rates for both A-like and B-like A states of GFPs upon deuterium exchange in Table 2 looks very suggestive, as it can be readily understood as the deuteron being more localized than the proton and thus showing a smaller $f$ (Section S8). Slight decreases in the Stark tuning rates of deuterated PYPs were also found as compared to the protonated counterparts (Figure 6B), lending support to this argument. However, by examining Figure 6A,C and viewing the GFP variant series as a whole rather than individually, the data points from deuterated samples do not follow a noticeably different trend in a comparison with the protonated counterparts, which is in contradiction with a smaller $f$ expected for deuterated species, so it appears that proton polarizability cannot explain the observed isotope effect. Rather, it could be plausibly rationalized as an electronic effect in response to the isotope substitution, the same origin as the electrostatic modulation of the chromophore's color. ${ }^{39,40}$ Specifically, the SIE can explain this observation: because deuteration causes a blue and red shift of A-like and B-like A states, respectively, ${ }^{36}$ it reduces the Stark tuning rates in both cases based on the Stark tuning rate vs absorption maximum correlation from the chromophores in their corresponding protonation states (Figure 6A,C). The degree of the Stark tuning rate decrease also correlates with the magnitude of the SIE (Table S10), further strengthening the plausibility of electrostatic modulation over proton polarizability. The anomalies in the observed Stark tuning rates from short-hydrogen-bond GFP variants can therefore be fully explicated with the covalency of the short hydrogen bond (Section 3.2.1), and the observed large $f \Delta \mu$ for PYPs can also be accounted for using a similar argument.

Even though it seems unlikely that the Zundel polarizability is a dominant mechanism for the perturbation from short hydrogen bonds in these proteins, it might be more prominent in systems with more delocalized protons, such as true FBHBs, where the anticipated polarization could be less masked by the substituents' electronic effect. ${ }^{76}$ Past evidence for the Zundel polarizability has relied heavily on vibrational observables through IR or Raman spectroscopy to avoid substantial electronic polarization from the probes, where the electric fields were provided by condensed-phase environments (e.g., solvents, ions, and protein residues) ${ }^{74}$ rather than externally applied. It would be very interesting to search for proteins with precalibrated vibrational probes ${ }^{45}$ participating in short hydrogen bonds and to directly apply an external field in order to validate the existence of proton polarizability through enhanced Stark tuning rates within these proteins.

\section{CONCLUSIONS}

Using the normal A and B states as well-calibrated electrostatic probes, we identify how the electron distribution of the chromophore is perturbed when engaging in a short hydrogen bond. This leads to a smaller Stark tuning rate for the protonated chromophore but enhances the Stark tuning rate for the deprotonated chromophores. The correlation between Stark tuning rates and absorption maxima obtained from these situations deviates 
from that expected from pure electrostatic color tuning. On the basis of the applicability of classical electrostatics, we are thus able to distinguish short hydrogen bonds from other noncovalent interactions, including normal hydrogen bonds, $\pi-\pi$ stacking, and cation/ anion $-\pi$ interactions. Specifically, electrons are still confined within the $\pi$ system of the chromophore when participating in noncovalent interactions, and this can be treated well with classical electrostatics. Conversely, covalent modifications, such as halogenation and short hydrogen bonds, allow electrons to smear outside the original conjugation through exchange interactions due to the quantum nature of electrons, and this is combined with the classical electrostatically induced electron polarization.

With a proton-sensitive electronic probe, we also demonstrate how either electronic Stark or preresonance Raman spectroscopy can be a useful diagnostic tool for LBHBs. We use these methods to reinforce our previous argument that short-hydrogen-bond GFPs do not contain LBHBs regardless of how closely we match the donor-acceptor $\mathrm{p} K_{a} \cdot{ }^{36} \mathrm{We}$ also observed no evidence to support the notion of large proton polarizabilities from shorthydrogen-bond GFPs and PYPs, likely because the proton(s) involved is not delocalized enough due to the absence of LBHBs. Given the ubiquity of short hydrogen bonds in proteins $^{22,23}$ in combination with the scarce examples characterized with LBHBs, ${ }^{22,27,28}$ there seems to be lack of an energetic advantage for LBHBs to exist, either due to the aforementioned symmetry breaking originating from solvation ${ }^{75}$ or the difficulty of stabilizing a delocalized charge within polar environments. ${ }^{77,78}$ Given the large proton polarizability hypothesized within these short hydrogen bonds, ${ }^{74}$ it is evident how the corresponding structure-energetics relationship is strongly context dependent, ${ }^{10,18,19,79-82}$ and the role of the environment is the key to resolving conflicting results for this longstanding problem. In this regard, more local vibrational probes could be more suitable for measuring the environmental subtleties ${ }^{45}$ and identifying the appropriate parameter(s) for quantifying different environments of short hydrogen bonds.

\section{Supplementary Material}

Refer to Web version on PubMed Central for supplementary material.

\section{ACKNOWLEDGMENTS}

\footnotetext{
We thank Professor Robert Stanley at Temple University for the Stark spectra fitting software and Professor Pakorn Tony Kanchanawong at National University of Singapore for his suggestions on the Stark spectroscopy setup. We thank Drs. Aina Cohen, Pete Dunten, Tzanko Doukov, and Irimpan Mathews at the Stanford Synchrotron Radiation Lightsource (SSRL) for technical assistance during X-ray data collection. We greatly appreciate the late Professor Noel Hush's and Professor Sharon Hammes-Schiffer's illuminating feedback on various related topics. We also thank Dr. Alan Deng for solving two crystal structures with fluorines; Dr. Henk Both for optimizing PYP expression protocols and providing some PYP samples; Dr. Matt Romei for developing the Raman methodology and reading the manuscript; Drs. Luke Oltrogge, Amr Tamimi, Jacek Kozuch, and Sam Schneider for their helpful suggestions; Tom Carver in the Stanford Nano Shared Facilities for depositing nickel on Stark windows; Nacho the cat for his useful whiskers during crystal seeding. C.-Y.L. was supported by a Kenneth and Nina Tai Stanford Graduate Fellowship and the Taiwanese Ministry of Education. This work was supported, in part, by NIH Grant GM118044 (to S.G.B.) and NSF CCI Phase I: Center for First-Principles Design of Quantum Processes (CHE-1740645). Use of SSRL, SLAC National Accelerator Laboratory, is supported by the U.S. Department of Energy, Office of Science, Office of Basic Energy Sciences under Contract No. DE-AC02-76SF00515. The SSRL Structural Molecular Biology Program is supported by the DOE Office of Biological and Environmental Research, and by the National Institutes of Health, National Institute of General Medical Sciences (including P41GM103393). The contents of this publication are solely the responsibility of the authors and do not necessarily represent the
} 
official views of NIGMS or NIH. Part of this work was performed at the Stanford Nano Shared Facilities (SNSF), supported by the National Science Foundation under award ECCS-1542152.

\section{REFERENCES}

(1). Jeffrey GA; Saenger W Hydrogen Bonding in Biological Structures; Springer-Verlag: New York, 1991.

(2). Steiner T The hydrogen bond in the solid state. Angew. Chem., Int. Ed 2002, 41, 48-76.

(3). Anslyn EV; Dougherty DA Modern Physical Organic Chemistry; University Science Books: Sausalito, CA, 2006; pp 145-204.

(4). Ishikita H; Saito K Proton transfer reactions and hydrogen-bond networks in protein environments. J. R. Soc., Interface 2014, 11, 20130518. [PubMed: 24284891]

(5). Pimentel GC; McClellan AL The Hydrogen Bond; Freeman WH: San Francisco, CA, 1960.

(6). Schuster P; Zundel G; Sandorfy C The Hydrogen Bond: Recent Developments in Theory and Experiments; North-Holland: Amsterdam, the Netherlands, 1976.

(7). Gilli G; Gilli P The Nature of the Hydrogen Bond: Outline of a Comprehensive Hydrogen Bond Theory; Oxford University Press: Oxford, UK, 2009.

(8). Smith DA A Brief History of the Hydrogen Bond. In Modeling the Hydrogen Bond; Smith DA, Ed.; ACS Symposium Series 569; American Chemical Society: Washington, DC, 1994; pp 1-5.

(9). Perrin CL Are short, low-barrier hydrogen bonds unusually strong? Acc. Chem. Res 2010, 43, 1550-1557. [PubMed: 20939528]

(10). Herschlag D; Pinney MM Hydrogen bonds: Simple after all? Biochemistry 2018, 57, 3338-3352. [PubMed: 29678112]

(11). Perrin CL; Nielson JB "Strong" hydrogen bonds in chemistry and biology. Annu. Rev. Phys. Chem 1997, 48, 511-544. [PubMed: 9348662]

(12). Gilli P; Pretto L; Bertolasi V; Gilli G Predicting hydrogen-bond strengths from acid-base molecular properties. The $\mathrm{p} K_{\mathrm{a}}$ slide rule: Toward the solution of a long-lasting problem. Acc. Chem. Res 2009, 42, 33-44. [PubMed: 18921985]

(13). McKenzie RH; Bekker C; Athokpam B; Ramesh SG Effect of quantum nuclear motion on hydrogen bonding. J. Chem. Phys 2014, 140, 174508. [PubMed: 24811647]

(14). Sobczyk L; Grabowski SJ; Krygowski TM Interrelation between H-bond and $\pi$-electron delocalization. Chem. Rev 2005, 105, 3513-3560. [PubMed: 16218560]

(15). Fried SD; Boxer SG Thermodynamic framework for identifying free energy inventories of enzyme catalytic cycles. Proc. Natl. Acad. Sci. U. S. A 2013, 110, 12271-12276. [PubMed: 23840058]

(16). Ceriotti M; Fang W; Kusalik PG; McKenzie RH; Michaelides A; Morales MA; Markland TE Nuclear quantum effects in water and aqueous systems: Experiment, theory, and current challenges. Chem. Rev 2016, 116, 7529-7550. [PubMed: 27049513]

(17). Perrin CL Symmetries of hydrogen bonds in solution. Science 1994, 266, 1665-1668. [PubMed: 17775625]

(18). Nadal-Ferret M; Gelabert R; Moreno M; Lluch JM Are there really low-barrier hydrogen bonds in proteins? The case of photoactive yellow protein. J. Am. Chem. Soc 2014, 136, 3542-3552. [PubMed: 24548066]

(19). Graen T; Inhester L; Clemens M; Grubmüller H; Groenhof G The low barrier hydrogen bond in the photoactive yellow protein: A vacuum artifact absent in the crystal and solution. J. Am. Chem. Soc 2016, 138, 16620-16631. [PubMed: 27966904]

(20). Thomson B; Both J; Wu Y; Parrish RM; Martínez TJ; Boxer SG Perturbation of short hydrogen bonds in photoactive yellow protein via noncanonical amino acid incorporation. J. Phys. Chem. B 2019, 123, 4844-4849. [PubMed: 31117606]

(21). Meot-Ner (Mautner), M. Update 1 of: Strong ionic hydrogen bonds. Chem. Rev 2012, 112, PR22-PR103. [PubMed: 22873941]

(22). Hosur MV; Chitra R; Hegde S; Choudhury RR; Das A; Hosur RV Low-barrier hydrogen bonds in proteins. Crystallogr. Rev 2013, 19, 3-50. 
(23). Zhou S; Wang L Unraveling the structural and chemical features of biological short hydrogen bonds. Chem. Sci 2019, 10, 7734-7745. [PubMed: 31588321]

(24). Cleland WW; Kreevoy MM Low-barrier hydrogen bonds and enzymic catalysis. Science 1994, 264, 1887-1890. [PubMed: 8009219]

(25). Gerlt JA; Kreevoy MM; Cleland WW; Frey PA Understanding enzymic catalysis: The importance of short, strong hydrogen bonds. Chem. Biol 1997, 4, 259-267. [PubMed: 9195866]

(26). Yamaguchi S; Kamikubo H; Kurihara K; Kuroki R; Niimura N; Shimizu N; Yamazaki Y; Kataoka M Low-barrier hydrogen bond in photoactive yellow protein. Proc. Natl. Acad. Sci. U. S. A 2009, 106, 440-444. [PubMed: 19122140]

(27). Agback P; Agback T Direct evidence of a low barrier hydrogen bond in the catalytic triad of a serine protease. Sci. Rep 2018, 8, 10078. [PubMed: 29973622]

(28). Dai S; Funk L-M; von Pappenheim FR; Sautner V; Paulikat M; Schröder B; Uranga J; Mata RA; Tittmann K Low-barrier hydrogen bonds in enzyme cooperativity. Nature 2019, 573, 609-613. [PubMed: 31534226]

(29). Kreevoy MM; Liang T; Chang K-C Structures and isotopic fractionation factors of complexes AHA-1. J. Am. Chem. Soc 1977, 99, 5207-5209.

(30). Gilli P; Bertolasi V; Ferretti V; Gilli G Covalent nature of the strong homonuclear hydrogen bond. Study of the O-H-O system by crystal structure correlation methods. J. Am. Chem. Soc 1994, 116, 909-915.

(31). Gilli G; Gilli P Towards an unified hydrogen-bond theory. J. Mol. Struct 2000, 552, 1-15.

(32). Hansen PE; Spanget-Larsen J NMR and IR investigations of strong intramolecular hydrogen bonds. Molecules 2017, 22, 552.

(33). Feyer V; Prince KC; Coreno M; Melandri S; Maris A; Evangelisti L; Caminati W; Giuliano BM; Kjaergaard HG; Carravetta V Quantum effects for a proton in a low-barrier, double-well potential: Core level photoemission spectroscopy of acetylacetone. J. Phys. Chem. Lett 2018, 9, 521-526. [PubMed: 29314844]

(34). Shu X; Kallio K; Shi X; Abbyad P; Kanchanawong P; Childs W; Boxer SG; Remington SJ Ultrafast excited-state dynamics in the green fluorescent protein variant S65T/H148D. 1. Mutagenesis and structural studies. Biochemistry 2007, 46, 12005-12013. [PubMed: 17918959]

(35). Shi X; Abbyad P; Shu X; Kallio K; Kanchanawong P; Childs W; Remington SJ; Boxer SG Ultrafast excited-state dynamics in the green fluorescent protein variant S65T/H148D. 2. Unusual photophysical properties. Biochemistry 2007, 46, 12014-12025. [PubMed: 17918960]

(36). Oltrogge LM; Boxer SG Short hydrogen bonds and proton delocalization in green fluorescent protein (GFP). ACS Cent. Sci 2015, 1, 148-156. [PubMed: 27162964]

(37). Bublitz GU; Boxer SG Stark spectroscopy: Applications in chemistry, biology, and materials science. Annu. Rev. Phys. Chem 1997, 48, 213-242. [PubMed: 9348658]

(38). Silverman LN; Kanchanawong P; Treynor TP; Boxer SG Stark spectroscopy of mixed-valence systems. Philos. Trans. R. Soc., A 2008, 366, 33-45.

(39). Lin C-Y; Romei MG; Oltrogge LM; Mathews II; Boxer SG Unified model for photophysical and electro-optical properties of green fluorescent proteins. J. Am. Chem. Soc 2019, 141, 1525015265. [PubMed: 31450887]

(40). Lin C-Y; Boxer SG Mechanism of color and photoacidity tuning for the protonated green fluorescent protein chromophore. J. Am. Chem. Soc 2020, 142, 11032-11041. [PubMed: 32453950]

(41). Anderson S; Crosson S; Moffat K Short hydrogen bonds in photoactive yellow protein. Acta Crystallogr., Sect. D: Biol. Crystallogr 2004, D60, 1008-1016.

(42). Saito K; Ishikita H Energetics of short hydrogen bonds in photoactive yellow protein. Proc. Natl. Acad. Sci. U. S. A 2012, 109, 167-172. [PubMed: 22173632]

(43). Weinhold F; Landis CR Valency and Bonding: A Natural Bond Orbital Donor-Acceptor Perspective, 1st ed.; Cambridge University Press: Cambridge, UK, 2005; pp 593-661.

(44). Hunt PA; Ashworth CR; Matthews RP Hydrogen bonding in ionic liquids. Chem. Soc. Rev 2015, 44, 1257-1288. [PubMed: 25582457] 
(45). Fried SD; Boxer SG Measuring electric fields and noncovalent interactions using the vibrational Stark effect. Acc. Chem. Res 2015, 48, 998-1006. [PubMed: 25799082]

(46). Goeltz JC; Kubiak CP Mixed valency across hydrogen bonds. J. Am. Chem. Soc 2010, 132, 17390-17392. [PubMed: 21087020]

(47). Porter TM; Heim GP; Kubiak CP Stable mixed-valent complexes formed by electron delocalization across hydrogen bonds of pyrimidinone-linked metal clusters. J. Am. Chem. Soc 2018, 140, 12756-12759. [PubMed: 30265005]

(48). Getzoff ED; Gutwin KN; Genick UK Anticipatory active-site motions and chromophore distortion prime photoreceptor PYP for light activation. Nat. Struct. Mol. Biol 2003, 10, 663668.

(49). Sugishima M; Tanimoto N; Soda K; Hamada N; Tokunaga F; Fukuyama K Structure of photoactive yellow protein (PYP) E46Q mutant at 1.2 A resolution suggests how Glu46 controls the spectroscopic and kinetic characteristics of PYP. Acta Crystallogr., Sect. D: Biol. Crystallogr 2004, D60, 2305-2309.

(50). Brudler R; Meyer TE; Genick UK; Devanathan S; Woo TT; Millar DP; Gerwert K; Cusanovich MA; Tollin G; Getzoff ED Coupling of hydrogen bonding to chromophore conformation and function in photoactive yellow protein. Biochemistry 2000, 39, 13478-13486. [PubMed: 11063584]

(51). Memmi S; Kyndt J; Meyer T; Devreese B; Cusanovich M; Van Beeumen J Photoactive yellow protein from the halophilic bacterium Salinibacter ruber. Biochemistry 2008, 47, 2014-2024. [PubMed: 18198898]

(52). Kaledhonkar S; Hara M; Stalcup TP; Xie A; Hoff WD Strong ionic hydrogen bonding causes a spectral isotope effect in photoactive yellow protein. Biophys. J 2013, 105, 2577-2585. [PubMed: 24314088]

(53). Oltrogge LM; Wang Q; Boxer SG Ground-state proton transfer kinetics in green fluorescent protein. Biochemistry 2014, 53, 5947-5957. [PubMed: 25184668]

(54). Premvardhan LL; van der Horst MA; Hellingwerf KJ; van Grondelle R Stark spectroscopy on photoactive yellow protein, E46Q, and a nonisomerizing derivative, probes photo-induced charge motion. Biophys. J 2003, 84, 3226-3239. [PubMed: 12719252]

(55). Gurusaran M; Shankar M; Nagarajan R; Helliwell JR; Sekar K Do we see what we should see? Describing non-covalent interactions in protein structures including precision. IUCrJ 2014, 1, 74-81.

(56). Haddad KC; Sudmeier JL; Bachovchin DA; Bachovchin WW $a$-Lytic protease can exist in two separately stable conformations with different $\mathrm{His}^{57}$ mobilities and catalytic activities. Proc. Natl. Acad. Sci. U. S. A 2005, 102, 1006-1011. [PubMed: 15657134]

(57). Treynor TP Non-Classical Stark Effects of Photosynthetic Reaction Centers. Ph.D. Dissertation, Stanford University, Stanford, CA, 2003; pp 193-207.

(58). Bell AF; He X; Wachter RM; Tonge PJ Probing the ground state structure of the green fluorescent protein chromophore using Raman spectroscopy. Biochemistry 2000, 39, 4423-4431. [PubMed: 10757992]

(59). He X; Bell AF; Tonge PJ Isotopic labeling and normal-mode analysis of a model green fluorescent protein chromophore. J. Phys. Chem. B 2002, 106, 6056-6066.

(60). Fried SD; Boxer SG Evaluation of the evaluation of the energetics of the concerted acid-base mechanism in enzyme catalysis: The case of ketosteroid isomerase. J. Phys. Chem. B 2012, 116, 690-697. [PubMed: 22148842]

(61). Olah GA The $\sigma$-bridged 2-norbornyl cation and its significance to chemistry. Acc. Chem. Res 1976, 9, 41-52.

(62). Olah GA; Prakash GKS; Saunders M Conclusion of the classical-nonclassical ion controversy based on the structural study of the 2-norbornyl cation. Acc. Chem. Res 1983, 16, 440-448.

(63). von R. Schleyer P; Mainz VV; Strom ET Norbornyl Cation Isomers Still Fascinate. In The Foundations of Physical Organic Chemistry: Fifty Years of the James Flack Norris Award; Strom ET, Mainz VV, Eds.; ACS Symposium Series 1209; American Chemical Society: Washington, DC, 2015; pp 139-168. 
(64). Kanchanawong P; Dahlbom MG; Treynor TP; Reimers JR; Hush NS; Boxer SG Charge delocalization in the special-pair radical cation of mutant reaction centers of Rhodobacter sphaeroides from Stark spectra and nonadiabatic spectral simulations. J. Phys. Chem. B 2006, 110, 18688-18702. [PubMed: 16970500]

(65). Crutchley RJ Intervalence charge transfer and electron exchange studies of dinuclear ruthenium complexes. Adv. Inorg. Chem 1994, 41, 273-325.

(66). Londergan $\mathrm{CH}$; Kubiak CP Electron transfer and dynamic infrared-band coalescence: It looks like dynamic NMR spectroscopy, but a billion times faster. Chem. - Eur. J 2003, 9, 5962-5969. [PubMed: 14679508]

(67). Day P; Hush NS; Clark RJH Mixed valence: Origins and developments. Philos. Trans. R. Soc., A 2008, 366, 5-14.

(68). Marder SR; Gorman CB; Meyers F; Perry JW; Bourhill G; Brédas J-L; Pierce BM A unified description of linear and nonlinear polarization in organic polymethine dyes. Science 1994, 265 , 632-635. [PubMed: 17752759]

(69). Pinney MM; Natarajan A; Yabukarski F; Sanchez DM; Liu F; Liang R; Doukov T; Schwans JP; Martínez TJ; Herschlag D Structural coupling throughout the active site hydrogen bond networks of ketosteroid isomerase and photoactive yellow protein. J. Am. Chem. Soc 2018, 140, 9827-9843. [PubMed: 29990421]

(70). Arnold WD; Oldfield E The chemical nature of hydrogen bonding in proteins via NMR: J-couplings, chemical shifts, and AIM theory. J. Am. Chem. Soc 2000, 122, 12835-12841.

(71). Fried SD; Bagchi S; Boxer SG Extreme electric fields power catalysis in the active site of ketosteroid isomerase. Science 2014, 346, 1510-1514. [PubMed: 25525245]

(72). Wang L; Fried SD; Boxer SG; Markland TE Quantum delocalization of protons in the hydrogenbond network of an enzyme active site. Proc. Natl. Acad. Sci. U. S. A 2014, 111, 18454-18459. [PubMed: 25503367]

(73). McConnell HM Theory of nuclear magnetic shielding in molecules: I. Long-range dipolar shielding of protons. J. Chem. Phys 1957, 27, 226-229.

(74). Zundel G Hydrogen Bonds with Large Polarizability and Proton Transfer Processes in Electrochemistry and Biology. In Advances in Chemical Physics; Prigogine, I, Rice SA, Eds.; John Wiley \& Sons, Inc.: New York, 2000; Vol. 111, pp 1-217.

(75). Perrin CL; Lau JS Hydrogen-bond symmetry in zwitterionic phthalate anions: Symmetry breaking by solvation. J. Am. Chem. Soc 2006, 128, 11820-11824. [PubMed: 16953621]

(76). Janoschek R; Weidemann EG; Pfeiffer H; Zundel G Extremely high polarizability of hydrogen bonds. J. Am. Chem. Soc 1972, 94, 2387-2896.

(77). Warshel A; Papazyan A; Kollman PA On low-barrier hydrogen bonds and enzyme catalysis. Science 1995, 269, 102-106. [PubMed: 7661987]

(78). Warshel A; Papazyan A Energy considerations show that low-barrier hydrogen bonds do not offer a catalytic advantage over ordinary hydrogen bonds. Proc. Natl. Acad. Sci. U. S. A 1996, 93, 13665-13670. [PubMed: 8942991]

(79). Scheiner S; Redfern P; Szczęśniak MM Effects of external ions on the energetics of proton transfers across hydrogen bonds. J. Phys. Chem 1985, 89, 262-266.

(80). Trommsdorff HP Localized and delocalized proton levels in hydrogen bonds: Fluorescence line narrowing and Stark effect measurements. J. Lumin 1987, 38, 129-133.

(81). Bala P; Lesyng B; Truong TN; McCammon JA Ab initio studies and quantum-classical molecular dynamics simulations for proton transfer processes in model systems and in enzymes. In NATO Advanced Science Institutes Series C Vol. 368: Molecular Aspects of Biotechnology: Computational Models and Theories; Bertmn J, Ed.; Kluwer Academic Publishers: Dordrecht, The Netherlands, 1992; pp 299-326.

(82). Sigala PA; Ruben EA; Liu CW; Piccoli PMB; Hohenstein EG; Martinez TJ; Schultz AJ; Herschlag D Determination of hydrogen bond structure in water versus aprotic environments to test the relationship between length and stability. J. Am. Chem. Soc 2015, 137, 5730-5740. [PubMed: 25871450] 

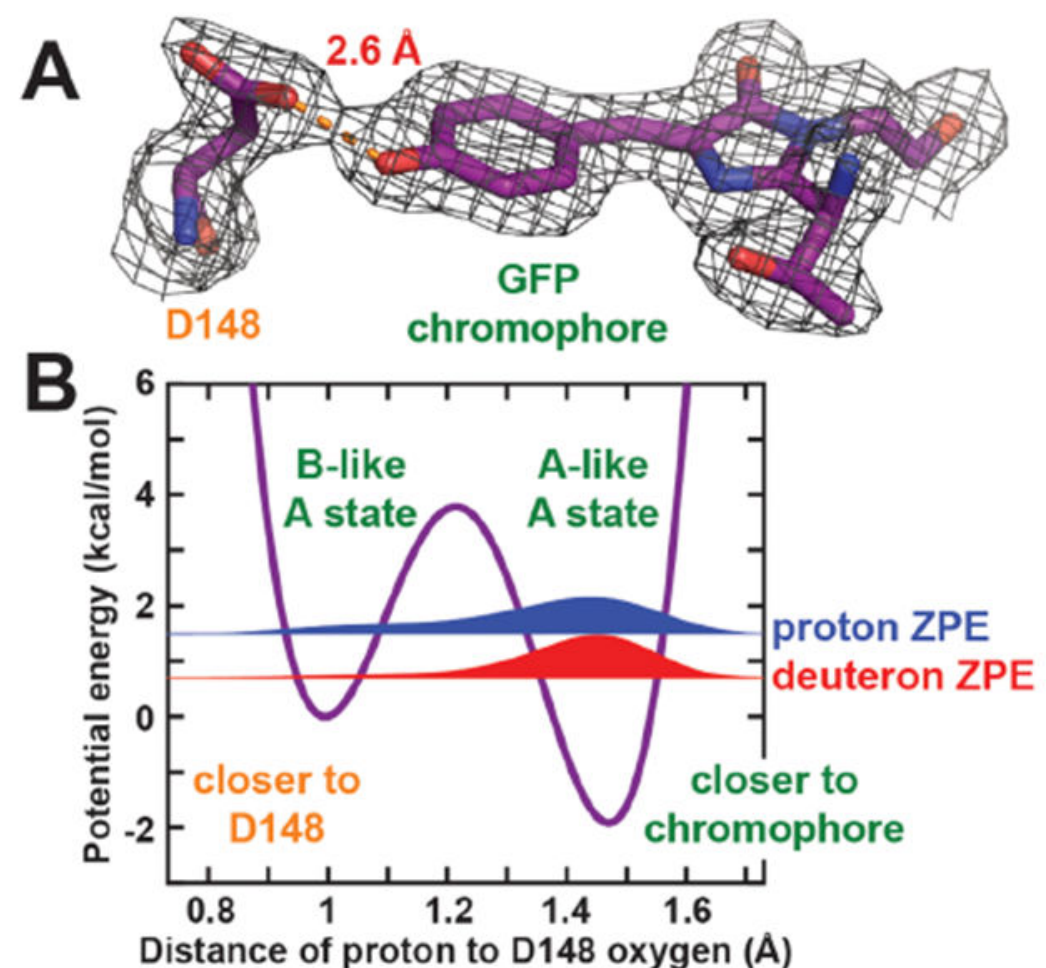<smiles>[X]c1cc(/C=C2\N=C(CC)N(C/C=C/C(C)C(=O)OCOc3ccc(/C=C4\N=C(CC)N(C)C4=O)cc3)C2=O)ccc1OOOC(=O)C(C)C</smiles>

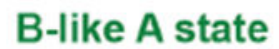

A-like A state

C<smiles>CC(C)c1ccc(O)cc1</smiles><smiles>C1CCCCC1</smiles><smiles>CCc1cccc(F)c1</smiles><smiles>Oc1ccc(I)cc1Cl</smiles><smiles>CC(C)(C)CCc1ccc(O)c(F)c1F</smiles><smiles>CCc1cc(F)c(O)c(F)c1</smiles><smiles>CCc1cc(Cl)c(O)c(I)c1</smiles>

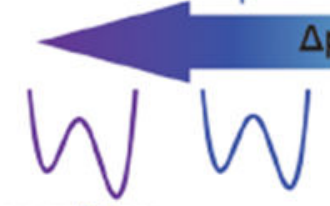

positive

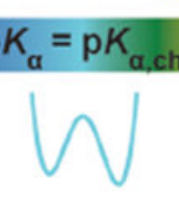

W

\section{matched}

negative

\section{Figure 1.}

Structure and energetics of the short hydrogen bond in ih:GFP S65T H148D variants (see Section $\mathrm{S} 1$ for the nomenclature of circularly permuted GFPs). (A) Chromophore and D148 structure with the electron density $\left(2 m F_{\mathrm{O}}-D F_{\mathrm{c}}\right.$ contoured at $\left.1 \sigma\right)$ of ih:GFP S65T H148D (PDB: 4ZF3 ${ }^{36}$ ). Structures of other variants can be found in Section S4 and figures therein; chromophore-D148 O-O distances for other variants are listed in Table 1. (B) A representative proton PES (in this case the Y66 variant) calculated from McKenzie's onedimensional coupled Morse potential mode ${ }^{13}$ with parameters determined in the previous 
study. ${ }^{36}$ The relative free energy between the two wells is governed by $\Delta \mathrm{p} K_{a}$ (vide infra) through $\Delta G^{\circ}=R T \ln 10 \Delta \mathrm{p} K_{a}$, where $T$ is at $300 \mathrm{~K}$. The two energy wells correspond to the proton residing more at the chromophore or at D148, which we refer to as the A-like and B-like A states, respectively. The corresponding ground-state wave function and ZPE for proton or deuteron are shown in blue or red, respectively. Deuteration lowers the ZPE and reduces delocalization. (C) The relative proton affinity, $\Delta \mathrm{p} K_{a}$, of the short hydrogen bond is tuned via systematic introduction of halogen(s) to the chromophore in the H148D background, and the estimated PESs are shown below the arrow. $\Delta \mathrm{p} K_{a}$ values for the variants are listed in Table 1. 

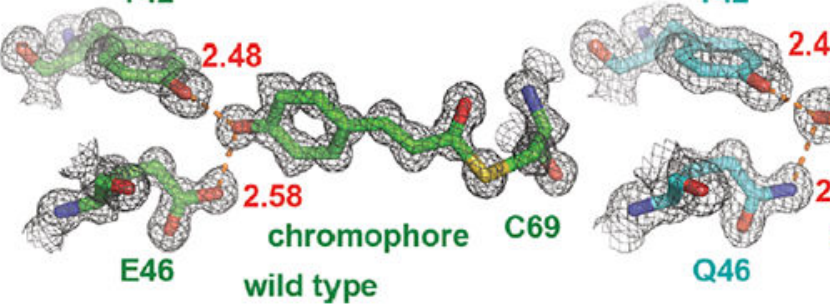

chromophore

C69

E46Q

2.51 chromophore C69

\section{Figure 2.}

Local structure with the electron density $\left(2 m F_{\mathrm{O}}-D F_{\mathrm{c}}\right.$ contoured at $\left.1 \sigma\right)$ for each HhPYP mutant, including wild-type (green, left, PDB: $1 \mathrm{NWZ}^{48}$ ), E46Q (cyan, middle, PDB: $1 \mathrm{UGU}^{49}$ ), and $\mathrm{Y} 42 \mathrm{~F}$ (magenta, right, $\mathrm{PDB}: 1 \mathrm{~F} 9 \mathrm{I}^{50}$ ). The hydrogen bond distances (in $\AA$ ) are labeled in red and also listed in Table 1. 

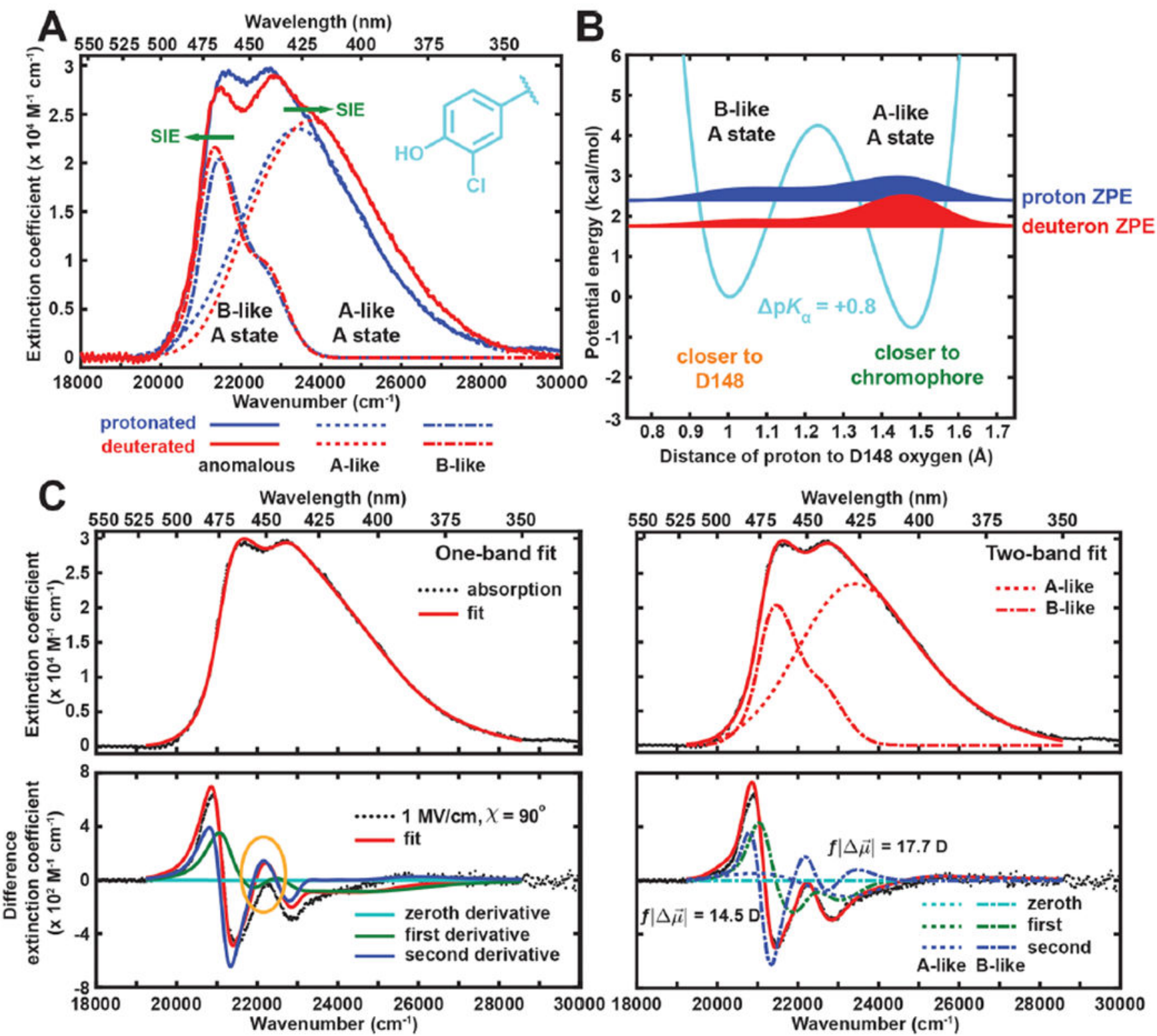

Figure 3.

Absorption spectra, Stark spectra, and energetics of a representative S65T H148D GFP variant, ih:GFP S65T Y66 $\left(3-\mathrm{Cl}_{1} \mathrm{Y}\right) \mathrm{H} 148 \mathrm{D}$. (A) The $77 \mathrm{~K}$ absorption spectra of the protonated (blue) and deuterated (red) species at $\mathrm{pH} 5$ and $\mathrm{pD} 5$, respectively. The corresponding A-like (dashed) and B-like (dashed-dotted) A state bands are deconvolved from simultaneous fitting of the absorption and Stark spectra (panel C). The direction of SIE upon deuteration for each underlying population is shown with a green arrow. Note that the maximum extinction coefficient of the normal protonated chromophore in GFP is about $60 \%$ of the deprotonated counterpart. ${ }^{53}$ (B) The corresponding PES and ZPEs for the short hydrogen bond with $\Delta \mathrm{p} K_{a}=+0.8$. The color coding follows Figure 1B. Deuteration further localizes the hydron wave function toward the donor and acceptor, causing the SIE seen in panel A. (C) The $77 \mathrm{~K}$ absorption (upper panels) and $2 \omega$ Stark spectra (lower panels, scaled 
to $1 \mathrm{MV} / \mathrm{cm}$ ) for the protonated $\mathrm{Y} 66\left(3-\mathrm{Cl}_{1} \mathrm{Y}\right) / \mathrm{H} 148 \mathrm{D}$ variant at $\mathrm{pH} 5$. The sum-of-derivative analysis is performed with one-band (left panels) and two-band (right panels) fits. One can see that the one-band fit is not satisfactory for both spectra simultaneously, especially in the region around $22000 \mathrm{~cm}^{-1}$ (circled), so an additional set of Stark parameters is required to fully recapitulate both band shapes. The Stark tuning rates in Table 2 can then be extracted from the magnitudes of the second-derivative components. ${ }^{37}$ 


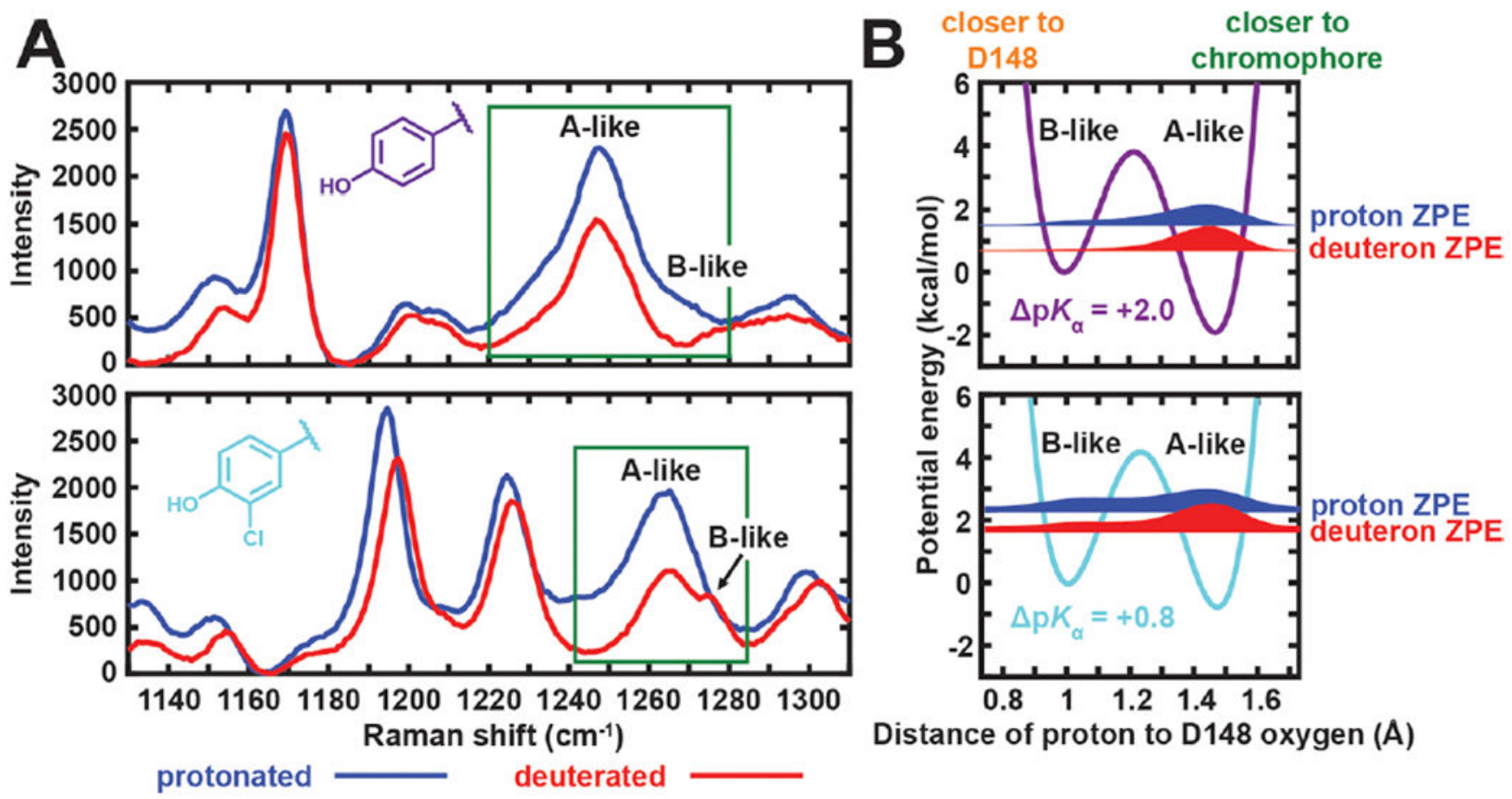

Figure 4.

Preresonance Raman spectra and derived PESs for $\mathrm{p} K_{a}$-matched GFPs. (A) Preresonance Raman spectra with $633 \mathrm{~nm}$ excitation of representative protonated (pH 5, blue traces) and deuterated (pD 5, red traces) S65T H148D GFP variants, which are Y66 (top) and $\mathrm{Y} 66\left(3-\mathrm{Cl}_{1} \mathrm{Y}\right)$ (bottom) in this case. The peaks of interest, corresponding to a proton-sensitive phenol stretching mode, are highlighted within green boxes. Raman features outside the boxes are associated with other phenol modes that are not protonation-sensitive. ${ }^{59}$ Note that the observed Raman intensities are not concentration normalized. The appearances of the Raman bands can be understood through (B) their corresponding PESs and ZPEs, which are reproduced from Figures 1B and 3B. More discussion can be found in Section S6. 

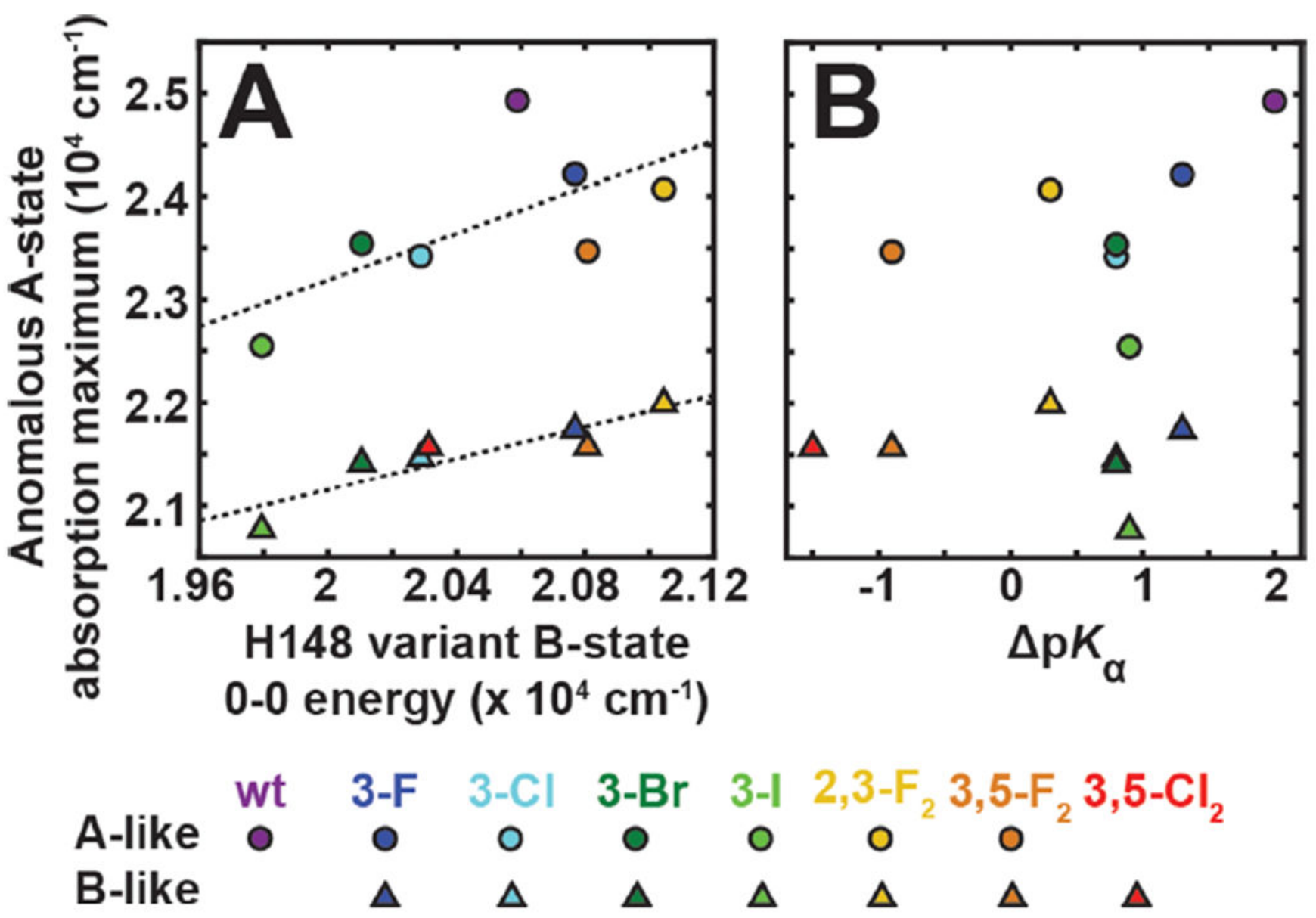

Figure 5.

Correlation plots of the absorption maxima from the anomalous A states (Table S10) with

(A) B-state 0-0 energy from their corresponding ih:GFP S65T H148 variants (Table S11) and (B) $\Delta \mathrm{p} K_{a}$ (Table 1). The color coding is consistent with Figure 1C. The plots are meant to gauge the contribution of electronic and proton position effects from halogenation to the absorption maxima of the anomalous A state. The electronic effect shows substantial influences on both the A-like and B-like A states, while the former is much more sensitive to the proton effect. 

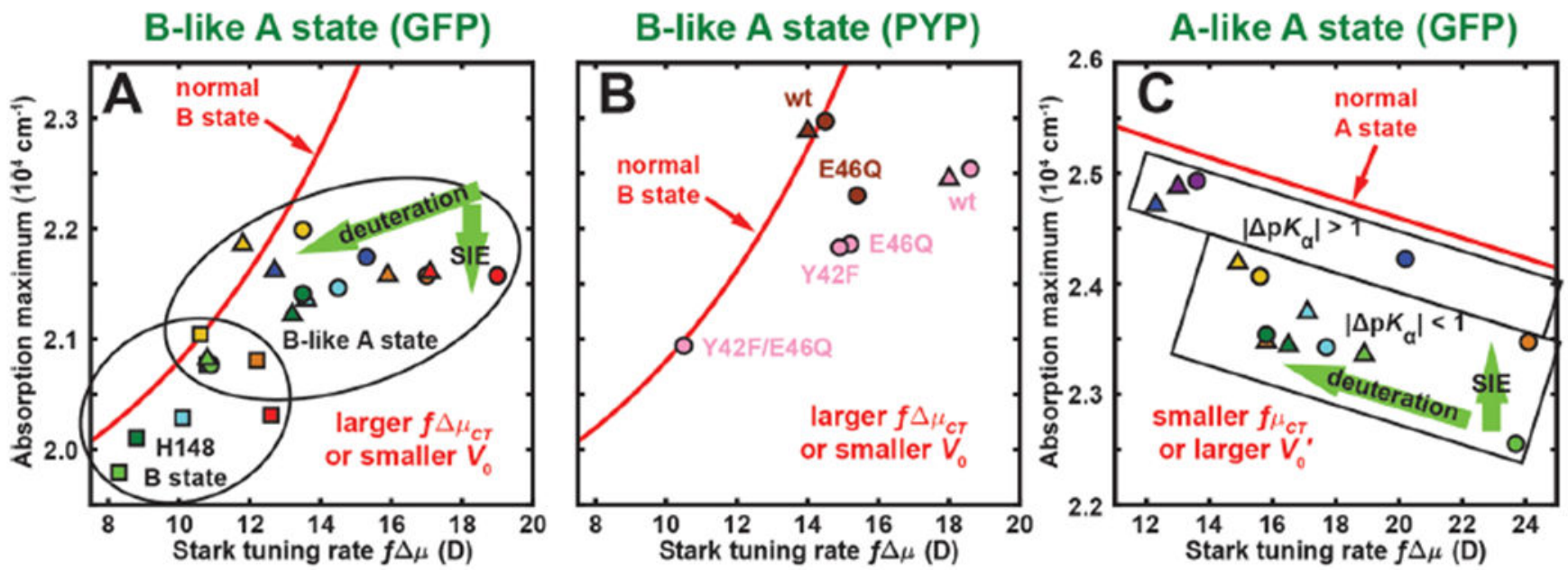

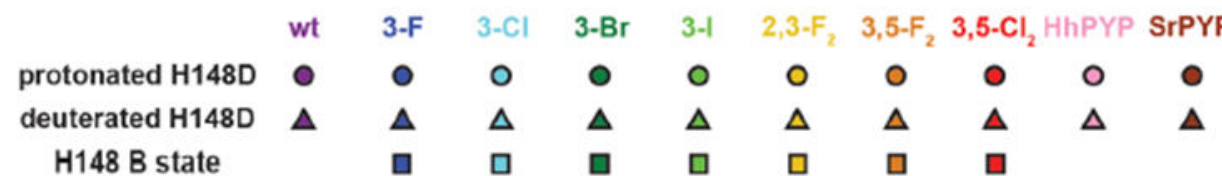

D

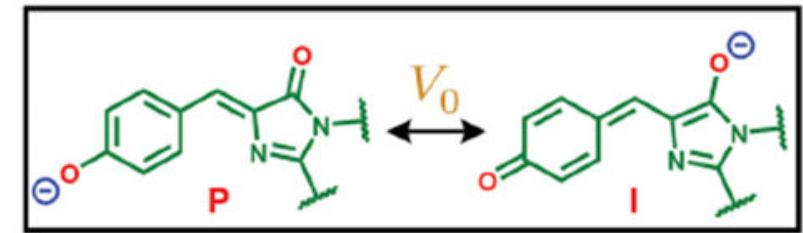

diabatic difference dipole $=\Delta \mu_{C T}$

$\mathbf{E}$
A state

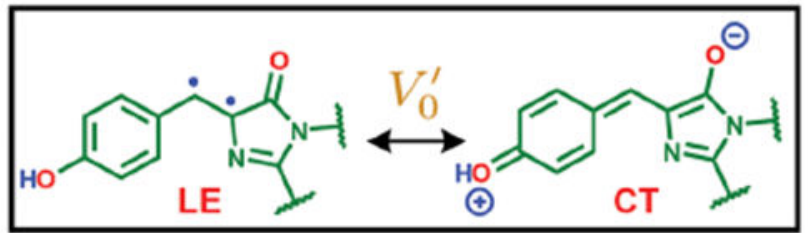

diabatic difference dipole $=\mu_{C T}$

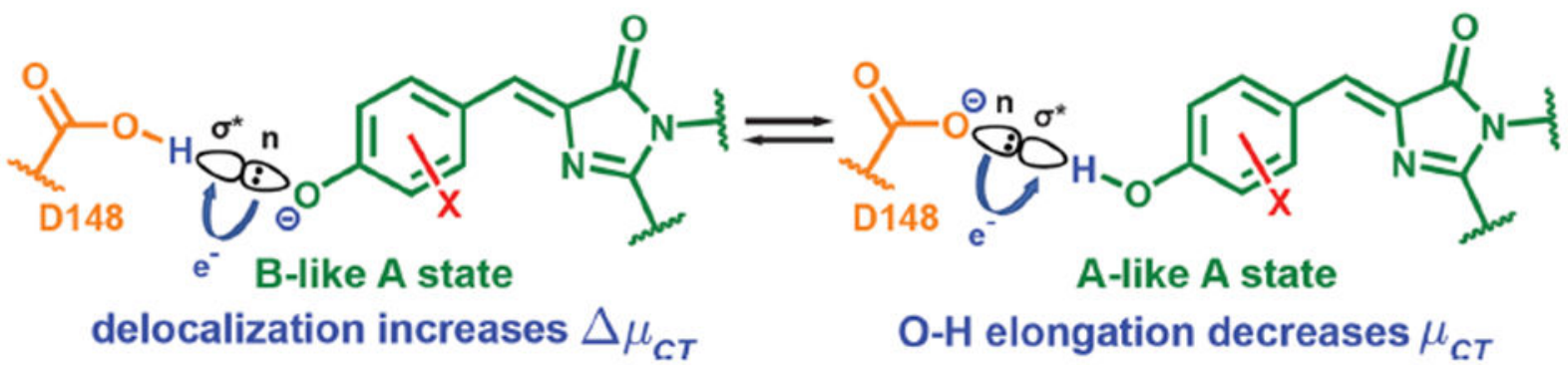

Figure 6.

Correlation of absorption maxima and Stark tuning rates and contributing resonance forms for (A) B-like A state from short-hydrogen-bond GFP variants, (B) PYP mutants, and (C) A-like A state from short-hydrogen-bond GFP variants (Table 2 and Table S10). The calibration curves for the corresponding normal states are reproduced from refs 39 and 40 and are shown in red. The trends of Stark tuning rate change and spectral shift (SIE) upon deuteration are represented with green thick arrows. (D) The diabatic difference dipole moments and couplings between the underlying resonance forms of the deprotonated (left) ${ }^{39}$ and protonated (right) chromophore. ${ }^{40}$ Technically, difference dipole moments matter for both protonation states. However, to better discern the two corresponding quantities, we drop 
the " $\Delta$ " for that of the protonated chromophore (right) as the LE form is insensitive to its electrostatic environment. ${ }^{40}$ (E) The molecular orbital picture of the short hydrogen bond within each anomalous A state, involving donation of electrons (shown as a lone pair) from the proton acceptor's nonbonding orbital (n) to the donor-H's $\sigma^{*}$ orbital through overlap. 


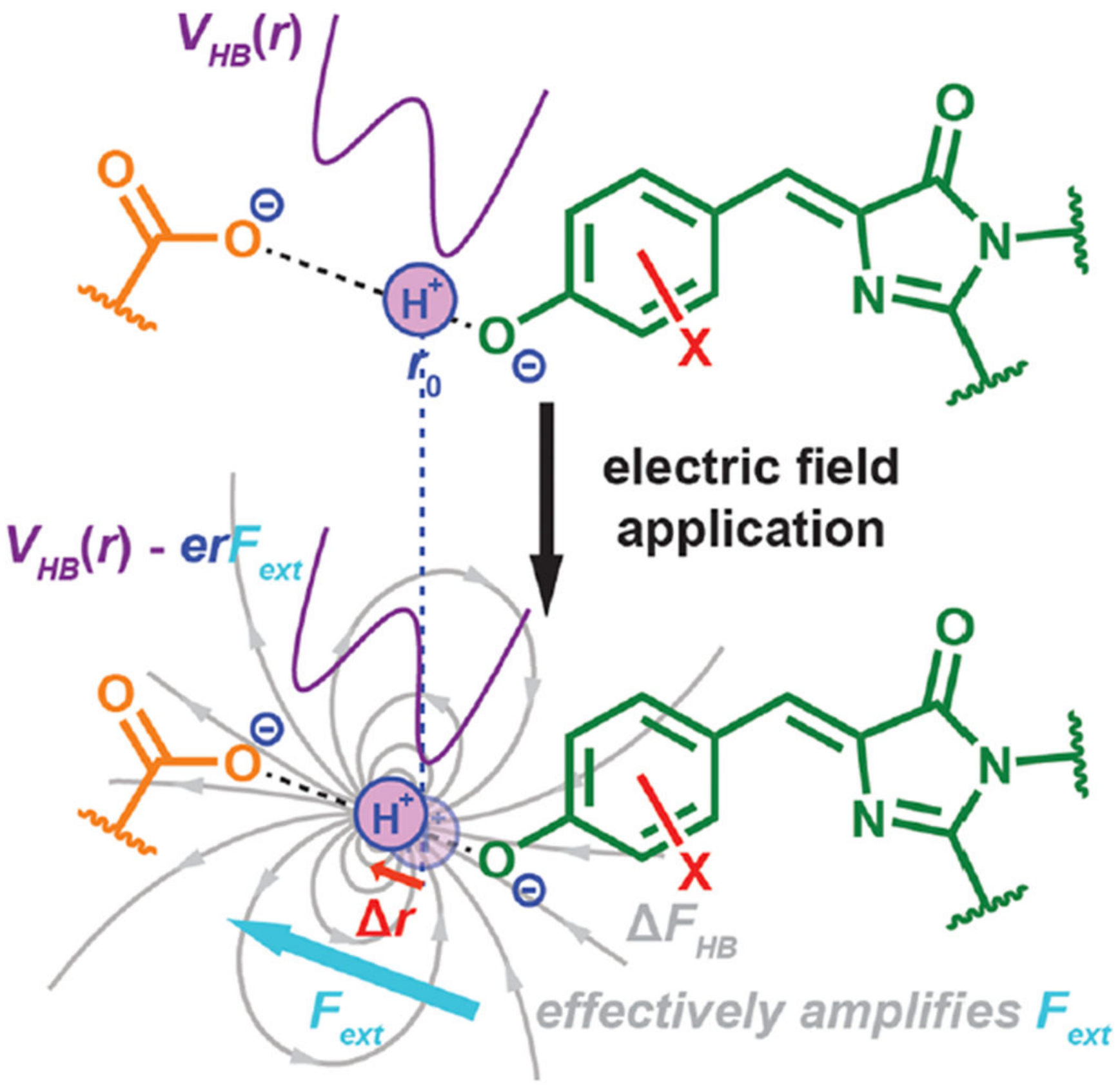

Figure 7.

Proton polarization within the short hydrogen bond (Zundel polarizability) may lead to an increase in the local electric field sensed by the chromophore. Without the external field (top), the equilibrium position of the proton is $r_{0}$ according to the proton PES $V_{\mathrm{HB}}(r)$ (purple curve). The externally applied field $F_{\text {ext }}$ (cyan arrow) displaces the proton (bottom, displacement exaggerated) by perturbing the PES and thereby induces a dipole field $\Delta F_{\mathrm{HB}}$ (gray field lines). Since the chromophore experiences both $F_{\text {ext }}$ and $\Delta F_{\mathrm{HB}}$ in the same direction, the proton polarization effectively amplifies the external field and consequently 
contributes to the local field factor $f$. Since shorter hydrogen bonds are expected to possess shallower PESs, the corresponding Zundel polarizability should be appreciable. 

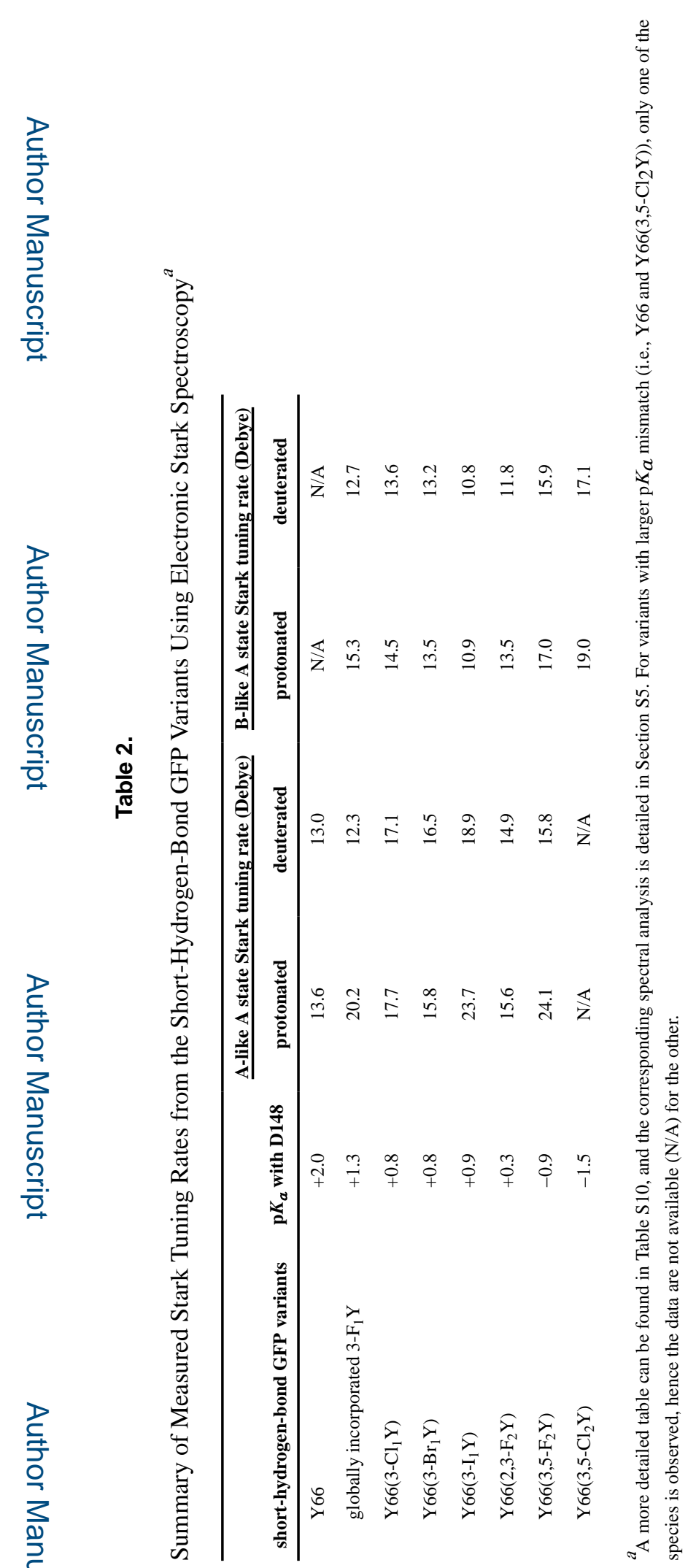